\title{
A Tripartite Game Analysis of Environmental Pollution Control Based on Complicated Intergovernmental Relations
}

\author{
Ling Li, ${ }^{1}$ Yao Song $\mathbb{D}^{2}{ }^{2}$ and Zhiqiang Zhang ${ }^{3}$ \\ ${ }^{1}$ Business School, Tianjin University of Finance and Economics, Tianjin 300222, China \\ ${ }^{2}$ Great Wall Enterprise Institute (Tianjin), Tianjin 300384, China \\ ${ }^{3}$ College of Management and Economics, Tianjin University, Tianjin 300072, China
}

Correspondence should be addressed to Yao Song; songyao@gei.com.cn

Received 12 May 2021; Accepted 8 August 2021; Published 23 August 2021

Academic Editor: Lei Xie

Copyright (C) 2021 Ling Li et al. This is an open access article distributed under the Creative Commons Attribution License, which permits unrestricted use, distribution, and reproduction in any medium, provided the original work is properly cited.

\begin{abstract}
An excellent ecological environment is conducive for improving economic benefits and social benefits. The environmental pollution control requires the cooperation of governments at all levels and a large amount of capital investment. However, under the system with Chinese characteristics, the intergovernmental relations present complex and dynamic characteristics: the central government is authoritative, the local governments are obedient and self-interested, and the environmental pollution control usually involves multiple government departments, while it has strong externality, which makes it easy to breed "free rider" behavior in the process of environmental pollution control. Therefore, the cross-regional environmental pollution control cooperation model of governments at all levels is a complex and worthwhile research problem. Based on this, the paper studies a tripartite game problem of environmental pollution control from both horizontal and vertical intergovernmental relations. The Hamilton-Jacobi-Bellman equation was used to obtain the optimal effort strategy, environmental pollution control level, and environmental pollution losses under the Nash game model, the Stackelberg game model, and the Cooperative game model. The results show the following: firstly, the governments' environmental pollution control efforts are positively related to their own execution ability and influence ability and negatively related to the cost coefficient; secondly, from the perspectives of environmental pollution control level and environmental pollution losses, the Cooperative game model is superior to the Nash game model and the Stackelberg game; thirdly, this paper analyzes the relationship between the loss-bearing ratio, the special financial funds, the effort level of government, and the environmental pollution control level; finally, the conclusions are verified by numerical analysis, which proves the validity of the models.
\end{abstract}

\section{Introduction}

With the acceleration of globalization and regional economic integration, China's regional environmental pollution problems have become increasingly prominent, showing obvious regional characteristics [1-3]. The deterioration of the overall regional environmental quality is a severe threat to public health and economic development. It is tough to control the spread of pollutants, as the ecological environment is increasingly showing obvious integration characteristics, and a single local government is often unable to solve complex regional environmental problems. Therefore, as an effective form of environmental protection, cross-regional cooperation in environmental governance has received widespread attention [4]. However, due to the externality of environmental pollution control and the division of administrative regions, cross-border environmental disputes between various administrative jurisdictions in China have gradually increased. Therefore, how to effectively regulate trans-boundary environmental pollution has always been an important issue in environmental protection.

Under China's traditional performance evaluation system and fiscal decentralization system, the competition goal of local governments is mainly the growth of regional GDP, which has an adverse effect on environmental pollution and environmental governance, and an impact on the regional ecological environment through the negative externality of 
environmental pollution and the positive externality of environmental governance. In terms of environmental pollution, local governments are overly pursuing economic growth while neglecting the ecological environment, and even sacrificing the environment in exchange for short-term economic growth, leading to environmental pollution. For example, local governments have adopted fiscal and tax preferential measures to attract certain companies that pollute, causing environmental pollution in the region to increase. The environmental pollution has negative externality, which can affect the environmental conditions of neighboring areas through transfer and diffusion, resulting in the worsening of pollution in neighboring areas. In terms of environmental governance, local governments often squeeze environmental protection expenditure due to excessive emphasis on local economic construction, resulting in insufficient investment in ecological environmental governance. Environmental governance has positive externality. When local governments increase local environmental protection and pollution control efforts, neighboring areas can freely enjoy the results of environmental governance through free-riding. In addition, the financial competition of local governments can easily derive local protectionist behaviors, hinder the realization of cross-regional environmental governance cooperation, and cause the negative externality of environmental pollution to become more obvious. At the same time, it also reduces the positive externality of environmental governance.

In order to achieve better governance results, coordination and cooperation between all levels of government must be strengthened. This is the realistic basis for this paper. There is no shortage of such examples in China. In the revision, we have added two typical examples of government cooperation in the treatment of environmental pollution. The details are as follows:

(1) As a highly systematic natural geographic unit of surface water, the watershed is inevitably divided by different administrative regions, which has spawned a contradiction between the integrity of the watershed and the division of administrative divisions. As a result, a series of disputes over the trans-boundary treatment of water pollution have emerged. The Yellow River flows through nine provinces (autonomous regions) of China, including Qinghai, Sichuan, Gansu, Ningxia, Inner Mongolia, Shaanxi, Shanxi, Henan, and Shandong. It flows into the Bohai Sea in Shandong Province and flows through a vast area. Most of the key industrial enterprises in the upper and middle reaches of the Yellow River Basin rely on energy advantages to build, including petrochemical and metallurgical enterprises. Although these companies have vigorously promoted the rapid economic and social development, they have also caused an imbalance in the industrial layout and frequent safety accidents of some companies, leading to the occurrence of major water pollution incidents. The coordinated management of water pollution in the Yellow River Basin has received great attention from the state. In September 2019, Xi Jinping personally deployed at the Symposium on Ecological Protection and High-Quality Development of the Yellow River Basin, elevated the ecological protection and high-quality development of the Yellow River Basin to a major national strategy, and pointed out that "the Yellow River Basin is governed by protection and governance." At the same time, it requires "coordinated promotion of large-scale governance"; in December of the same year, Xi Jinping delivered an important speech in the "Seeking Truth" magazine, in which he stated that ecological protection and high-quality development of the Yellow River Basin should be an important part of regional coordinated development.

(2) The Beijing-Tianjin-Hebei region is the most affected area in my country where air pollution is discharged continuously and superimposed across administrative regions. According to the " 2020 China Ecological and Environmental Status Bulletin" issued by the Ministry of Ecology and Environment, the air quality in Shijiazhuang, Tangshan, and Handan in the Beijing-Tianjin-Hebei region is poor. The percentage of days with good air quality in Beijing-Tianjin-Hebei and in the " $2+26$ " surrounding cities is $63.5 \%$. As a typical developed urban agglomeration in my country, the Beijing-Tianjin-Hebei region began to explore crossadministrative cooperative air pollution control methods relatively early and gained certain experience. At present, the Beijing-Tianjin-Hebei Air Pollution Prevention and Control Team has been established, and its members include "seven provinces and regions, eight ministries and commissions" (The seven provinces and regions include: Beijing, Tianjin, Hebei, Shanxi, Shandong, Henan, and Inner Mongolia; the ministries and commissions include: the Environmental Protection Agency, the Development and Reform Commission, the Ministry of Industry and Information Technology, the Ministry of Finance, the Ministry of Housing and Urban-rural Development, the Bureau of Meteorology, the $\mathrm{Bu}-$ reau of Energy, and the Ministry of Transport). The air pollution prevention and control coordination mechanism of Beijing-Tianjin-Hebei and surrounding areas has been successfully established, which provides a mode for the cooperation of governments at all levels in environmental governance across administrative regions.

Under the system with Chinese characteristics, the central government is authoritative in vertical intergovernmental relations, ensuring that the central government effectively controls and leads local governments. China's traditional fiscal decentralization system and the promotion mechanism of local government officials also determine the versatility of government functions. Local governments obey 
the leadership of the central government and accept supervision.

Therefore, based on the externality of the environmental pollution control, the authority of the central government, and the obedience and self-interest of local governments, this paper constructs a tripartite game between the central government and dual local governments to discuss the optimizing strategy of capital investment in the environmental pollution control under intergovernmental relations. This is of great significance for clarifying as to how to coordinate and promote environmental protection among governments at all levels in the future.

The theoretical significance, practical contribution, and innovation of the paper are as follows:

(1) The theoretical significance: taking the cross-administrative environmental issues as the research category, the benefit analysis as the research perspective, and the differential game as the research method, this paper is based on the intergovernmental relationship in environmental pollution control, and builds the benefit game and coordination analysis framework of the cross-administrative environmental governance. In addition, this paper conducts an in-depth analysis of the benefit games and conflicts behind noncooperative behaviors among governments at all levels, and proposes a path for constructing benefit coordination mechanisms in cross-administrative environmental governance, which can deeply reveal the general laws of regional public governance. This has certain theoretical significance for enriching and perfecting the theory of regional public governance.

(2) The practical contribution: facing the inefficiency of cross-administrative ecological environment governance, this paper takes the cross-administrative environmental governance as the starting point, and analyzes how the central government and local governments cooperate and the benefit games that appear in the cooperation. The research conclusions of this paper are of great significance to effectively solve the environmental problems of cross-administrative regions and realize the coordinated development of regions. At the same time, they also provide a reference for the mode of government cooperation in various fields such as politics, economy, culture, and society. It is conducive for promoting the sustained and healthy development of the entire national economy.

(3) The innovation: there are two main limitations in the existing research on the coordinated governance of environmental pollution between governments: (1) Most scholars adopt static game and staged game models, and do not consider the continuous changes of state variables and the amount of change in the strategies of the participants as the state changes; (2) existing studies are mostly two-party games. However, the actual participants in cross-regional environmental governance are generally three parties or even multiple parties. Relying on the differential game theory, in order to find the internal factors that affect the cooperation of all parties, this paper constructs a tripartite game model between the central government and the two local governments, discusses resource allocation and financial special funding strategies in environmental pollution control, and analyzes the endogenous reasons and paths of cooperation between the parties.

\section{Literature Review}

2.1. The Government Competition. Chirinko and Wilson [5] used the strategic tax competition theory to analyze how changes in capital tax policies in neighboring jurisdictions affect capital tax policies in a given jurisdiction for the USA. Perdiguero and Jiménez's research showed that the local competition, technical difficulties, and government competition were the main factors affecting the introduction of bio-diesel into the Spanish gasoline market [6]. Xu's research found that regional government competition was conducive for stimulating local governments to initiate and implement market-oriented reform activities, which reduced the occurrence of corruption to a certain extent [7]. Lin et al.'s research found that corporate political capital played an important but negative role in the cooperation of green product and the process of innovation performance [8]. Kubick and Masli's study found that government competition affected the provision and consumption of public goods. The positive impact of government competition on economic growth can make local governments take risky behavior [9]. Yu et al. [10] studied the spatial effects of prefecture-level cities in China and found that after excluding other factors such as economic spillovers and tax competition, competition among government leaders at the same level is the most important factor affecting the spatial effects. Hong and Lee [11] analyzed the policy differences of 47 cities in the USA and found that political competition will affect the government's supervision of the sharing economy. The government cared more about the benefit of market players than that of the public. Shi and $\mathrm{Xi}$ [12] found that competition between local governments may have a positive effect under certain conditions. The performance appraisal under the leadership of the central government had promoted spatial competition in safety governance and enlarged the intensity of safety supervision. This may be an important factor leading to a significant improvement in coal mine production safety in recent years. Gang et al. [13] used the random boundary model to calculate the green total factor productivity of 278 provinces and cities in China from 2004 to 2013. The study found an inverted U-shaped relationship between county-level government competition and green total factor productivity, and excessive cross-jurisdictional competition has an adverse effect on green total factor productivity (GTFP), while moderate government competition does not. Deng et al. [14] studied the impact of political competition through a game theory model and 
found that political competition will affect the best green technology innovation strategic model and the optimal investment ratio of environmental governance of local governments and enterprises. Wu [15] found that although the competition among local governments is not the main driving force for the formation of the industrial structure and division in the Yangtze River Delta, its role cannot be underestimated. Deng et al. [16] used super-efficiency data envelopment analysis to test the impact of local government competition and environmental regulation intensity on regional innovation performance and regional heterogeneity. At the same time, local governments compete for foreign direct investment (FDI) to participate in regional innovative production.

2.2. The Government's Role in the Environment. The positive effects: Fairchild [17] believed that increasing government subsidies to green production enterprises can effectively improve the clean production behavior of enterprises and indirectly reduce environmental pollution. Qiu and Yang [18] believed that the government plays an important coordinating role in environmental protection, and the implementation of the emission permit trading system is conducive for compensating the environmental protection costs of upstream companies and can promote the realization of Pareto optimal. Mir et al. [19] studied the relationship between government competition and environmental information disclosure in New Zealand, and the results showed that intensified political competition is conducive for increasing environmental information disclosure. Through the analysis of data from 29 provinces and cities in China, $\mathrm{Li}$ and $\mathrm{He}$ [20] studied the interaction between regional competition, environmental taxes, and green technology innovation. The results show that the influence of regional competition on green technology innovation also presents an "inverted U" shape, that is, benign regional competition is conducive for green technology innovation, but excessive regional competition produces the opposite result. Eichner and Pethig [21] analyzed countries with liquidity and local cross-border environmental pollution and found that competition between government emission taxes and capital taxes will have a significant impact on environmental pollution. Through the research on environmental issues in the Guangdong-Hong Kong-Macao Greater Bay Area, Ren [22] found that coordinated governance and administrative supervision between governments at all levels is an important driving force for promoting the construction of an environmental protection system. Tang and Qin [23] analyzed the impact of local government competition on green total factor productivity (GTFP) and its internal mechanism by using the SDM model and the mediating effect model.

The negative effects: Cremer's research on US emissions taxes found that the greater the pressure of political competition, the lower the willingness of local governments to implement high environmental taxes [24]. Qi and Zhang [25] have studied the relationship between the central government and local governments in China. The research shows that in order to attract more external investment and promote the development of the local economy, local governments tend to sacrifice the environment and protect environmentally polluting enterprises. Fedyukin and Igor's research showed that the existence of political competition will make local governments pay more attention to economic development to a certain extent, reduce their attention to environmental protection, and lead to aggravation of local environmental pollution [26].

2.3. The Game of Cross-Regional Government. Yu [27] drew on the experience of the European Union and the Netherlands in the treatment of water pollution, and on this basis, he studied the problem of trans-boundary water pollution in China. Kim et al. [28] designed a set of games with externality and connections to solve cross-regional water resources management problems. Fernandez [29] analyzed the problem of cross-border water pollution control in North America through the establishment of a game theory model. The research results show that cooperative games with water monitoring and information sharing decision-making are beneficial to reduce pollution costs and pollution damage. $\mathrm{Li}$ et al. [30] established a model to determine the comprehensive control strategy of cross-regional lake pollution in China based on environmental green costs, and the research results provided a theoretical basis for the formulation of emission permit prices.

\subsection{The Fiscal Expenditure and Decentralization Theory.} Brueckner and Jan [31] believed that when a fiscal policy has positive externality, it often leads to fiscal competition among local governments to reduce expenditures. This is the "free rider" psychology of local governments. The research of Wilson and Gordon [32] found that fiscal expenditure competition can benefit regional economic growth by improving the efficiency of fiscal fund use. $\mathrm{Xu}$ et al. [33] studied China's provincial panel data from 1995 to 2008 and found that there is a long-term equilibrium relationship between fiscal decentralization, local government competition, and cultivated land transfer. The research of Becker and Rauscher [34] believed that tax competition is conducive to economic growth by enhancing the mobility of factors. Prud'homme [35] pointed out that China's economic decentralization system has stimulated the effectiveness of local government's industrial decision-making to a certain extent, thereby promoting the development of strategic emerging industries, but these are inseparable from the central government's supervisory role. Skovgaard [36] found that environmental decision-making increasingly involves departments other than the environmental sector; especially, the Ministry of Finance has an increasingly important influence on environmental decision-making because they solve environmental problems by controlling the budget from a different perspective. Ercolano and Romano [37] studied the local fiscal environmental protection expenditures of European governments and found that the national local fiscal environmental protection expenditures are related to the 
country's development status. Bazavan. [38] found that China's decentralization system also allows local governments to play different roles in the process of economic development. They play the role of manager in some industries and the role of partner or investor in others.

\section{Model and Assumption}

This paper constructs a game model of environmental pollution control based on intergovernmental relationship. The model assumes that the environmental pollution control system consists of a central government (the abbreviation of a central government is Cent-gov), a local government $\mathrm{A}$ (the abbreviation of a local government A is Local-gov A), which is mainly affected by environmental pollution, and a local government $\mathrm{B}$ (the abbreviation of a local government $B$ is Local-gov B), which is secondarily affected by environmental pollution. When a sudden environmental pollution incident occurs, the city with the higher level of environmental pollution control can timely mobilize human, material, and financial resources; promptly organize emergency evacuation and resettlement of residents; and narrow the scope of environmental pollution. After the occurrence of environmental pollution, the central government and local governments will work together to verify the targets of assistance, transfer and resettle the victims, calculate the economic losses, improve the level of environmental pollution control, and carry out restoration and reconstruction work, in order to minimize environmental pollution losses.

In order to study the intergovernmental relationship, this paper makes the following variable hypotheses. $E_{a}(t)$ is the effort level of Local-gov $A$ in the process of environmental pollution control. $E_{b}(t)$ is the effort level of Localgov $B$ in the process of environmental pollution control. The effort level of the Cent-gov to improve the environmental pollution control level of Local-gov A and Local-gov B is $E_{a s}(t)$ and $E_{b s}(t)$, the environmental pollution control costs paid by the Cent-gov, Local-gov A, and Local-gov B are $C_{s}(t), C_{a}(t)$, and $C_{b}(t)$ :

$$
\begin{aligned}
& C_{s}(t)=\frac{\mu_{s}}{2}\left[E_{a s}^{2}(t)+E_{b s}^{2}(t)\right], \\
& C_{a}(t)=\frac{\mu_{a}}{2} E_{a}^{2}(t), \\
& C_{b}(t)=\frac{\mu_{b}}{2} E_{b}^{2}(t) .
\end{aligned}
$$

The environmental pollution control effort is directly proportional to the environmental pollution control costs, that is, as the environmental pollution control effort increases, the costs will also increase. $\mu_{s}, \mu_{a}$, and $\mu_{b}$, respectively, represent the cost coefficient of environmental pollution control by the Cent-gov, the Local-gov A, and the Local-gov B. $R_{a}(t), R_{b}(t)$, respectively, indicate the environmental pollution control level of the Local-gov $A$ and the Local-gov B at time $t$. The improvement of local government's environmental pollution control level stems from the joint efforts of the central government and the local governments. The improvement of environmental pollution control level has a natural decay law with time, and has the characteristics of cross-border and positive externality. Therefore, there is a certain spillover effect in the improvement of environmental pollution control level. The environmental pollution control level of Local-gov B is affected by Local-gov A. The changes of environmental pollution control level of the Local-gov A and the Local-gov $\mathrm{B}$ over time can be described by the following stochastic differential equations:

$$
\begin{aligned}
\frac{\mathrm{d} R_{a}(t)}{\mathrm{d} t}= & \alpha_{a} E_{a s}(t)+\beta_{a} E_{a}(t)-\gamma_{a} R_{a}(t) \\
\frac{\mathrm{d} R_{b}(t)}{\mathrm{d} t}= & \alpha_{b} E_{b s}(t)+\beta_{b} E_{b}(t)-\gamma_{b} R_{b}(t)+\eta \frac{\mathrm{d} R_{a}(t)}{\mathrm{d} t} \\
= & \eta\left[\alpha_{a} E_{a s}(t)+\beta_{a} E_{a}(t)-\gamma_{a} R_{a}(t)\right]+\alpha_{b} E_{b s}(t) \\
& +\beta_{b} E_{b}(t)-\gamma_{b} R_{b}(t) .
\end{aligned}
$$

The initial values of environmental pollution control level in the two regions: $R_{a}(0)=R_{a} \geq 0, R_{b}(0)=R_{b} \geq 0, \alpha$ represents the impact of the effort level of the Cent-gov on the local environmental pollution control level; $\beta$ represents the impact of the effort level of the local government on the local environmental pollution control level; $\gamma>0$ represents the degree of attenuation of environmental pollution control level; and $\eta \geq 0$ indicates the impact coefficient of Local-gov A's environmental pollution control level on Local-gov B's.

$L_{s}(t), L_{a}(t)$, and $L_{b}(t)$, respectively, represent the environmental pollution losses of the Cent-gov, Local-gov A, and Local-gov B at time $t . T_{s}(t), T_{a}(t)$, and $T_{b}(t)$, respectively, represent the total environmental pollution losses of the Cent-gov, Local-gov A, and Local-gov B at time $0-t$. At time $t$, the environmental pollution losses of the Localgov A and the Local-gov B are $L_{a}(t)$ and $L_{b}(t)$, respectively, which can be expressed as

$$
\begin{aligned}
& L_{a}(t)=M(t)-\varepsilon_{a} E_{a s}(t)-\delta_{a} E_{a}(t)-\theta_{a} R_{a}(t), \\
& L_{a}(t)=N(t)-\varepsilon_{b} E_{b s}(t)-\delta_{b} E_{b}(t)-\theta_{b} R_{b}(t),
\end{aligned}
$$

where $M, N$ are real numbers, indicating the maximum losses degree of the Local-gov A and the Local-gov B, $M>N$. $\varepsilon, \delta$ are constants greater than 0 , indicating the influence level of the Cent-gov's efforts and local government's efforts on environmental pollution losses, that is, the executive capacity of the Cent-gov and the local government. $\theta$ is the impact level of environmental pollution control level on environmental pollution losses.

\section{Game Strategy in Different Models}

According to different types of intergovernmental relations, environmental pollution control models can be divided into three types: the Nash game model, the Stackelberg game model, and the Cooperative game model. 
4.1. The Nash Game Model. The Nash game model is based on the natural division of labor, and the scope of interaction is small. The local government is decentralized, closed, and self-sufficient. Strictly speaking, this phenomenon does not exist, as local governments in this model, especially local governments with similar geographical areas, have no connection and communication. But, as power moves down, the central government has reduced the supervision of local governments. It is rare to actively engage in competition and cooperation strategies between local governments. The Local-gov A and the Local-gov B spontaneously carry out environmental pollution control, the Cent-gov does not subsidize local governments, and the three parties conduct noncooperative games. In the infinite time zone, all parties aim at minimizing their own environmental pollution losses, and they accordingly select the optimal effort level and make rational decisions. The environmental pollution losses are shared by the Cent-gov and local governments, the share ratio of the Cent-gov is $\omega_{i}\left(0 \leq \omega_{i} \leq 1, i=a, b\right)$, and the share ratio of local government is $1-\omega_{i}$. The Cent-gov and local government have the same positive discount rate $r$.

The objective function of Local-gov A can be expressed as

$$
T_{a}=\int_{0}^{\infty} e^{-r t}\left\{\left(1-\omega_{a}\right)\left[M(t)-\varepsilon_{a} E_{a s}(t)-\delta_{a} E_{a}(t)-\theta_{a} R_{a}(t)\right]+\frac{\mu_{a}}{2} E_{a}^{2}(t)\right\} \mathrm{d} t
$$

The objective function of Local-gov B can be expressed as

$$
T_{b}=\int_{0}^{\infty} e^{-r t}\left\{\left(1-\omega_{b}\right)\left[N(t)-\varepsilon_{b} E_{b s}(t)-\delta_{b} E_{b}(t)-\theta_{b} R_{b}(t)\right]+\frac{\mu_{b}}{2} E_{b}^{2}\right\} \mathrm{d} t .
$$

The objective function of Cent-gov can be expressed as

$$
T_{s}=\int_{0}^{\infty} e^{-r t}\left\{\begin{array}{c}
\omega_{a}\left[M(t)-\varepsilon_{a} E_{a s}(t)-\delta_{a} E_{a}(t)-\theta_{a} R_{a}(t)\right]+\frac{\mu_{a}}{2} E_{a s}^{2} \\
+\omega_{b}\left[N(t)-\varepsilon_{b} E_{b s}(t)-\delta_{b} E_{b}(t)-\theta_{b} R_{b}(t)\right]+\frac{\mu_{b}}{2} E_{b}^{2}
\end{array}\right\} \mathrm{d} t
$$

$E_{a s}(t), E_{b s}(t), E_{a}(t), E_{b}(t)$ are control variables, $R_{a}(t)$ and $R_{b}(t)$ are state variables. In addition to this, all other parameters are constants greater than 0 and not related to time. The optimal decision of each party is determined by the feedback control strategy. Since the parameters in the model are not related to time, the game subjects face the same game in the infinite time zone, and their strategies are static feedback equilibrium.

Proposition 1. In the Nash game model, the static feedback Nash equilibrium strategies of the Local-gov A, the Local-gov $B$, and the Cent-gov are

$$
\begin{aligned}
& E_{a}^{*}=\frac{1-\omega_{a}}{\mu_{a}}\left(\delta_{a}+\frac{\beta_{a} \theta_{a}}{r+\gamma_{a}}\right), \\
& E_{b}^{*}=\frac{1-\omega_{b}}{\mu_{b}}\left(\delta_{b}+\frac{\beta_{b} \theta_{b}}{r+\gamma_{b}}\right), \\
& E_{a s}^{*}=\frac{\omega_{a}}{\mu_{s}}\left(\varepsilon_{a}+\frac{\alpha_{a} \theta_{a}}{r+\gamma_{a}}\right)+\frac{\eta r \alpha_{a} \omega_{b} \theta_{b}}{\mu_{s}\left(r+\gamma_{a}\right)\left(r+\gamma_{b}\right)}, \\
& E_{b s}^{*}=\frac{\omega_{b}}{\mu_{s}}\left(\varepsilon_{b}+\frac{\alpha_{b} \theta_{b}}{r+\gamma_{b}}\right) .
\end{aligned}
$$


Proof. In order to obtain the Markov refined Nash equilibrium, it is assumed that there is a continuous bounded differential function $V_{i}\left(R_{a}, R_{b}\right) i \in(a, b, s)$ of environmental pollution losses, which satisfies the HJB (Hamilton-Jacobi-Bellman) equation for all $R_{a} \geq 0, R_{b} \geq 0$ :

$$
\begin{gathered}
r \cdot V_{a}\left(R_{a}, R_{b}\right)=\min _{E_{a} \geq 0}\left\{\begin{array}{c}
\left(1-\omega_{a}\right)\left(M-\varepsilon_{a} E_{a s}-\delta_{a} E_{a}-\theta_{a} R_{a}\right)+\frac{\mu_{a}}{2} E_{a}^{2} \\
-\left(\frac{\partial V_{a}}{\partial R_{a}}+\eta \frac{\partial V_{a}}{\partial R_{b}}\right)\left(\alpha_{a} E_{a s}+\beta_{a} E_{a}-\gamma_{a} R_{a}\right)-\frac{\partial V_{a}}{\partial R_{b}}\left(\alpha_{b} E_{b s}+\beta_{b} E_{b}-\gamma_{b} R_{b}\right)
\end{array}\right\}, \\
\left.\begin{array}{rl}
\left(1-\omega_{b}\right)\left(N-\varepsilon_{b} E_{a s}-\delta_{b} E_{a}-\theta_{b} R_{b}\right)+\frac{\mu_{b}}{2} E_{b}^{2} \\
V_{b}\left(R_{a}, R_{b}\right)=\min _{E_{b} \geq 0}\left\{\begin{array}{c}
\left.\frac{\partial V_{b}}{\partial R_{a}}+\eta \frac{\partial V_{b}}{\partial R_{b}}\right)\left(\alpha_{a} E_{a s}+\beta_{a} E_{a}-\gamma_{a} R_{a}\right)-\frac{\partial V_{b}}{\partial R_{b}}\left(\alpha_{b} E_{b s}+\beta_{b} E_{b}-\gamma_{b} R_{b}\right)
\end{array}\right\}, \\
r \cdot V_{s}\left(R_{a}, R_{b}\right)=\min _{E_{a s} \geq 0, E_{b s} \geq 0}\left\{\begin{array}{c}
\omega_{a}\left(M-\varepsilon_{a} E_{a s}-\delta_{a} E_{a}-\theta_{a} R_{a}\right)+\omega_{b}\left(N-\varepsilon_{b} E_{b s}-\delta_{b} E_{b}-\theta_{b} R_{b}\right)+\frac{\mu_{s}}{2}\left(E_{a s}^{2}+E_{b s}^{2}\right) \\
-\left(\frac{\partial V_{s}}{\partial R_{a}}+\eta \frac{\partial V_{s}}{\partial R_{b}}\right)\left(\alpha_{a} E_{a s}+\beta_{a} E_{a}-\gamma_{a} R_{a}\right)-\frac{\partial V_{s}}{\partial R_{b}}\left(\alpha_{b} E_{b s}+\beta_{b} E_{b}-\gamma_{b} R_{b}\right)
\end{array}\right.
\end{array}\right\} .
\end{gathered}
$$

By calculating the first-order partial derivatives of $E_{a}$ and $E_{b}$ for the right end of equations (11) and (12), and calculating the first-order partial derivatives of $E_{a s}$ and $E_{b s}$ by formula (13), and making them equal to zero, we can get

$$
\begin{aligned}
& E_{a}=\frac{\left(1-\omega_{a}\right) \delta_{a}+\beta_{a}\left(\left(\partial V_{a} / \partial R_{a}\right)+\eta\left(\partial V_{a} / \partial R_{b}\right)\right)}{\mu_{a}}, \\
& E_{b}=\frac{\left(1-\omega_{b}\right) \delta_{b}+\beta_{b}\left(\partial V_{b} / \partial R_{b}\right)}{\mu_{b}}
\end{aligned}
$$

$$
\begin{aligned}
& E_{a s}=\frac{\omega_{a} \varepsilon_{a}+\alpha_{a}\left(\left(\partial V_{s} / \partial R_{a}\right)+\eta\left(\partial V_{s} / \partial R_{b}\right)\right)}{\mu_{s}} \\
& E_{b s}=\frac{\omega_{b} \varepsilon_{b}+\alpha_{b}\left(\partial V_{s} / \partial R_{b}\right)}{\mu_{b}} .
\end{aligned}
$$

Substituting equations (14)-(17) into equations (11)-(13), we can get

$$
\begin{aligned}
r \cdot V_{a}= & \left.\gamma_{a}\left(\frac{\partial V_{a}}{\partial R_{a}}+\eta \frac{\partial V_{a}}{\partial R_{b}}\right)-\left(1-\omega_{a}\right) \theta_{a}\right] R_{a}+\gamma_{b} \frac{\partial V_{a}}{\partial R_{b}} R_{b} \\
& -\frac{\left[\omega_{a} \varepsilon_{a}+\alpha_{a}\left(\left(\partial V_{s} / \partial R_{a}\right)+\eta\left(\partial V_{s} / \partial R_{b}\right)\right)\right]\left[\left(1-\omega_{a}\right) \varepsilon_{a}+\alpha_{a}\left(\left(\partial V_{a} / \partial R_{a}\right)+\eta\left(\partial V_{a} / \partial R_{b}\right)\right)\right]}{\mu_{s}}-\frac{\alpha_{b}\left(\partial V_{a} / \partial R_{b}\right)\left(\omega_{b} \varepsilon_{b}+\alpha_{b}\left(\partial V_{s} / \partial R_{b}\right)\right)}{\mu_{s}} \\
& -\frac{\left[\left(1-\omega_{a}\right) \delta_{a}+\beta_{a}\left(\left(\partial V_{a} / \partial R_{a}\right)+\eta\left(\partial V_{a} / \partial R_{b}\right)\right)\right]^{2}}{2 \mu_{a}}-\frac{\beta_{b}\left(\partial V_{a} / \partial R_{b}\right)\left[\left(1-\omega_{b}\right) \delta_{b}+\beta_{b}\left(\partial V_{b} / \partial R_{b}\right)\right]}{2 \mu_{b}}+\left(1-\omega_{a}\right) M \\
r \cdot V_{b}= & \gamma_{a}\left(\frac{\partial V_{b}}{\partial R_{a}}+\eta \frac{\partial V_{b}}{\partial R_{b}}\right) R_{a}+\left[\gamma_{b} \frac{\partial V_{b}}{\partial R_{b}}-\left(1-\omega_{b}\right) \theta_{b}\right] R_{b} \\
& -\frac{\left(\omega_{b} \varepsilon_{b}+\alpha_{b}\left(\partial V_{s} / \partial R_{b}\right)\right)\left[\left(1-\omega_{b}\right) \varepsilon_{b}+\alpha_{b}\left(\partial V_{s} / \partial R_{b}\right)\right]}{\mu_{s}} \\
& -\frac{\alpha_{a}\left(\left(\partial V_{b} / \partial R_{a}\right)+\eta\left(\partial V_{b} / \partial R_{b}\right)\right)\left[\omega_{a} \varepsilon_{a}+\alpha_{a}\left(\partial V_{s} / \partial R_{a}+\eta\left(\partial V_{s} / \partial R_{b}\right)\right)\right]}{\mu_{s}} \\
& -\frac{\beta_{a}\left(\left(\partial V_{b} / \partial R_{a}\right)+\eta\left(\partial V_{b} / \partial R_{b}\right)\right)\left[\left(1-\omega_{a}\right) \delta_{a}+\beta_{a}\left(\left(\partial V_{a} / \partial R_{a}\right)+\eta\left(\partial V_{a} / \partial R_{b}\right)\right)\right]}{2 \mu_{a}} \\
& -\frac{\left[\left(1-\omega_{b}\right) \delta_{b}+\beta_{b}\left(\partial V_{b} / \partial R_{b}\right)\right]^{2}}{2 \mu_{b}}+\left(1-\omega_{b}\right) N
\end{aligned}
$$




$$
\begin{aligned}
r \cdot V_{s}= & {\left[\gamma_{a}\left(\frac{\partial V_{s}}{\partial R_{a}}+\eta \frac{\partial V_{s}}{\partial R_{b}}\right)-\omega_{a} \theta_{a}\right] R_{a}+\left(\gamma_{b} \frac{\partial V_{s}}{\partial R_{b}}-\omega_{b} \theta_{b}\right) R_{b}-\frac{\left[\omega_{a} \varepsilon_{a}+\alpha_{a}\left(\left(\partial V_{s} / \partial R_{a}\right)+\eta\left(\partial V_{s} / \partial R_{b}\right)\right)\right]^{2}}{2 \mu_{s}} } \\
& -\frac{\left(\omega_{b} \varepsilon_{b}+\alpha_{b}\left(\partial V_{s} / \partial R_{b}\right)\right)^{2}}{2 \mu_{s}}-\frac{\left[\left(1-\omega_{a}\right) \delta_{a}+\beta_{a}\left(\left(\partial V_{a} / \partial R_{a}\right)+\eta\left(\partial V_{a} / \partial R_{b}\right)\right)\right]\left[\omega_{a} \delta_{a}+\beta_{a}\left(\left(\partial V_{s} / \partial R_{a}\right)+\eta\left(\partial V_{s} / \partial R_{b}\right)\right)\right]}{\mu_{a}} \\
& -\frac{\left[\left(1-\omega_{b}\right) \delta_{b}+\beta_{b}\left(\partial V_{b} / \partial R_{b}\right)\right]\left(\omega_{b} \delta_{b}+\beta_{b}\left(\partial V_{s} / \partial R_{b}\right)\right)}{\mu_{b}}+\omega_{a} M+\omega_{b} N .
\end{aligned}
$$

From equations (18)-(20), it can be known that the linear optimal functions about $R_{a}, R_{b}$ are the solutions of the HJB equation. Let

$$
\begin{aligned}
& V_{a}\left(R_{a}, R_{b}\right)=p_{a} R_{a}+q_{a} R_{b}+u_{a}, \\
& V_{b}\left(R_{a}, R_{b}\right)=p_{b} R_{a}+q_{b} R_{b}+u_{b},
\end{aligned}
$$

$$
\begin{aligned}
r\left(p_{a} R_{a}+q_{a} R_{b}+u_{a}\right)= & {\left[\gamma_{a}\left(p_{a}+\eta q_{a}\right)-\left(1-\omega_{a}\right) \theta_{a}\right] R_{a}+\gamma_{b} q_{a} R_{b} } \\
& -\frac{\left[\omega_{a} \varepsilon_{a}+\alpha_{a}\left(p_{s}+\eta q_{s}\right)\right]\left[\left(1-\omega_{a}\right) \varepsilon_{a}+\alpha_{a}\left(p_{a}+\eta q_{a}\right)\right]}{\mu_{s}}-\frac{\alpha_{b} q_{a}\left(\omega_{b} \varepsilon_{b}+\alpha_{b} q_{s}\right)}{\mu_{s}} \\
& -\frac{\left[\left(1-\omega_{a}\right) \delta_{a}+\beta_{a}\left(p_{a}+\eta q_{a}\right)\right]^{2}}{2 \mu_{a}}-\frac{\beta_{b} q_{a}\left[\left(1-\omega_{b}\right) \delta_{b}+\beta_{b} q_{b}\right]}{2 \mu_{b}}+\left(1-\omega_{a}\right) M, \\
r\left(p_{b} R_{a}+q_{b} R_{b}+u_{b}\right)= & \gamma_{a}\left(p_{b}+\eta q_{b}\right) R_{a}+\left[\gamma_{b} q_{b}-\left(1-\omega_{b}\right) \theta_{b}\right] R_{b} \\
& -\frac{\left(\omega_{b} \varepsilon_{b}+\alpha_{b} g_{2}\right)\left[\left(1-\omega_{b}\right) \varepsilon_{b}+\alpha_{b} q_{b}\right]}{\mu_{s}}-\frac{\alpha_{a}\left(p_{b}+\eta q_{b}\right)\left[\omega_{a} \varepsilon_{a}+\alpha_{a}\left(p_{s}+\eta q_{s}\right)\right]}{\mu_{s}} \\
& -\frac{\beta_{a}\left(p_{b}+\eta q_{b}\right)\left[\left(1-\omega_{a}\right) \delta_{a}+\beta_{a}\left(p_{a}+\eta q_{a}\right)\right]}{2 \mu_{a}}-\frac{\left[\left(1-\omega_{b}\right) \delta_{b}+\beta_{b} q_{b}\right]^{2}}{2 \mu_{b}}+\left(1-\omega_{b}\right) N \\
r\left(p_{s} R_{a}+q_{s} R_{b}+u_{s}\right)= & {\left[\gamma_{a}\left(p_{s}+\eta q_{s}\right)-\omega_{a} \theta_{a}\right] R_{a}+\left(\gamma_{b} q_{s}-\omega_{b} \theta_{b}\right) R_{b} } \\
& -\frac{\left[\omega_{a} \varepsilon_{a}+\alpha_{a}\left(p_{s}+\eta q_{s}\right)\right]^{2}}{2 \mu_{s}}-\frac{\left(\omega_{b} \varepsilon_{b}+\alpha_{b} q_{s}\right)^{2}}{2 \mu_{s}}-\frac{\left[\left(1-\omega_{a}\right) \delta_{a}+\beta_{a}\left(p_{a}+\eta q_{a}\right)\right]\left[\omega_{a} \delta_{a}+\beta_{a}\left(p_{s}+\eta q_{s}\right)\right]}{\mu_{a}} \\
& -\frac{\left[\left(1-\omega_{b}\right) \delta_{b}+\beta_{b} q_{b}\right]\left(\omega_{b} \delta_{b}+\beta_{b} q_{s}\right)}{\mu_{b}}+\omega_{a} M+\omega_{b} N . \\
&
\end{aligned}
$$

If equations (24)-(26) satisfy all $R_{a} \geq 0, R_{b} \geq 0$, it is easy to get

$$
\begin{aligned}
& p_{a}=-\frac{\left(1-\omega_{a}\right) \theta_{a}}{r+\gamma_{a}}, \\
& p_{b}=0, \\
& u_{a}=-\frac{\left(1-\omega_{a}\right)\left[\varepsilon_{a}\left(r+\gamma_{a}\right)+\alpha_{a} \theta_{a}\right]\left\{\omega_{a}\left[\varepsilon_{a}\left(r+\gamma_{a}\right)+\alpha_{a} \theta_{a}\right]+\left(\eta r \alpha_{a} \omega_{b} \theta_{b} /\left(r+\gamma_{b}\right)\right)\right\}}{\mu_{s} r\left(r+\gamma_{a}\right)^{2}}-\frac{\left(1-\omega_{a}\right)^{2}\left[\delta_{a}\left(r+\gamma_{a}\right)+\beta_{a} \theta_{a}\right]^{2}}{2 \mu_{a} r\left(r+\gamma_{a}\right)^{2}}+\frac{\left(1-\omega_{a}\right) M}{r},
\end{aligned}
$$




$$
\begin{aligned}
p_{b}= & \frac{\lambda \gamma_{a}\left(1-\omega_{b}\right) \theta_{b}}{\left(r+\gamma_{a}\right)\left(r+\gamma_{b}\right)}, \\
q_{b}= & -\frac{\left(1-\omega_{b}\right) \theta_{b}}{r+\gamma_{b}}, \\
u_{b}= & -\frac{\eta \alpha_{a}\left(1-\omega_{b}\right) \theta_{b}\left\{\omega_{a}\left[\varepsilon_{a}\left(r+\gamma_{a}\right)+\alpha_{a} \theta_{a}\right]+\left(\eta r \alpha_{a} \omega_{b} \theta_{b} /\left(r+\gamma_{b}\right)\right)\right\}}{\mu_{s}\left(r+\gamma_{a}\right)^{2}\left(r+\gamma_{b}\right)} \\
& -\frac{\omega_{b}\left(1-\omega_{b}\right)\left[\varepsilon_{b}\left(r+\gamma_{b}\right)+\alpha_{b} \theta_{b}\right]^{2}}{\mu_{s} r\left(r+\gamma_{b}\right)^{2}}-\frac{\eta \beta_{a}\left(1-\omega_{b}\right) \theta_{b}\left(1-\omega_{a}\right)\left[\delta_{a}\left(r+\gamma_{a}\right)+\beta_{a} \theta_{a}\right]}{\mu_{a}\left(r+\gamma_{a}\right)^{2}\left(r+\gamma_{b}\right)} \\
& -\frac{\left(1-\omega_{b}\right)^{2}\left[\delta_{b}\left(r+\gamma_{b}\right)+\beta_{b} \theta_{b}\right]^{2}}{2 \mu_{b} r\left(r+\gamma_{b}\right)^{2}}+\frac{\left(1-\omega_{b}\right) N}{r}, \\
p_{s}= & \frac{\lambda \gamma_{a}\left(1-\omega_{b}\right) \theta_{b}}{\left(r+\gamma_{a}\right)\left(r+\gamma_{b}\right)}-\frac{\omega_{a} \theta_{a}}{r+\gamma_{a}}, \\
q_{s}= & -\frac{\omega_{2} \theta_{b}}{r+\gamma_{b}}, \\
u_{s}= & -\frac{\left\{\omega_{a}\left[\varepsilon_{a}\left(r+\gamma_{a}\right)+\alpha_{a} \theta_{a}\right]+\left(\eta r \alpha_{a} \omega_{b} \theta_{b} /\left(r+\gamma_{b}\right)\right)\right\}^{2}}{2 \mu_{s} r\left(r+\gamma_{a}\right)^{2}}-\frac{\omega_{b}^{2}\left[\varepsilon_{b}\left(r+\gamma_{b}\right)+\alpha_{b} \theta_{b}\right]^{2}}{2 \mu_{s} r\left(r+\gamma_{b}\right)^{2}}+\frac{\omega_{a} M+\omega_{b} N}{r} \\
& -\frac{\left(1-\omega_{a}\right)\left[\delta_{a}\left(r+\gamma_{a}\right)+\beta_{a} \theta_{a}\right]\left\{\omega_{a}\left[\delta_{a}\left(r+\gamma_{a}\right)+\beta_{a} \theta_{a}\right]+\left(\eta r \beta_{a} \omega_{b} \theta_{b} /\left(r+\gamma_{b}\right)\right)\right\}}{\mu_{a} r\left(r+\gamma_{a}\right)^{2}}-\frac{\omega_{b}\left(1-\omega_{b}\right)\left[\delta_{b}\left(r+\gamma_{b}\right)+\beta_{b} \theta_{b}\right]^{2}}{\mu_{b} r\left(r+\gamma_{b}\right)^{2}} .
\end{aligned}
$$

Substituting equations (27)-(29) into equations (21)-(23), we can obtain the minimum environmental pollution losses functions of Local-gov A, Local-gov A, and Cent-gov, as follows:

$$
\begin{aligned}
V_{a}^{*}= & \frac{\left(1-\omega_{a}\right) M}{r}-\frac{\left(1-\omega_{a}\right) \theta_{a}}{r+\gamma_{a}} R_{a}-\frac{\left(1-\omega_{a}\right)^{2}\left[\delta_{a}\left(r+\gamma_{a}\right)+\beta_{a} \theta_{a}\right]^{2}}{2 \mu_{a} r\left(r+\gamma_{a}\right)^{2}} \\
& -\frac{\left(1-\omega_{a}\right)\left[\varepsilon_{a}\left(r+\gamma_{a}\right)+\alpha_{a} \theta_{a}\right]\left\{\omega_{a}\left[\varepsilon_{a}\left(r+\gamma_{a}\right)+\alpha_{a} \theta_{a}\right]+\left(\eta r \alpha_{a} \omega_{b} \theta_{b} /\left(r+\gamma_{b}\right)\right)\right\}}{\mu_{s} r\left(r+\gamma_{a}\right)^{2}},
\end{aligned}
$$

$$
\begin{aligned}
V_{b}^{*}= & \frac{\left(1-\omega_{b}\right) N}{r}+\frac{\eta \gamma_{a}\left(1-\omega_{b}\right) \theta_{b}}{\left(r+\gamma_{a}\right)\left(r+\gamma_{b}\right)} R_{a}-\frac{\left(1-\omega_{b}\right) \theta_{b}}{r+\gamma_{b}} R_{b} \\
& -\frac{\eta \alpha_{a}\left(1-\omega_{b}\right) \theta_{b}\left\{\omega_{a}\left[\varepsilon_{a}\left(r+\gamma_{a}\right)+\alpha_{a} \theta_{a}\right]+\left(\eta r \alpha_{a} \omega_{b} \theta_{b} /\left(r+\gamma_{b}\right)\right)\right\}}{\mu_{s}\left(r+\gamma_{a}\right)^{2}\left(r+\gamma_{b}\right)}-\frac{\omega_{b}\left(1-\omega_{b}\right)\left[\varepsilon_{b}\left(r+\gamma_{b}\right)+\alpha_{b} \theta_{b}\right]^{2}}{\mu_{s} r\left(r+\gamma_{b}\right)^{2}} \\
& -\frac{\eta \beta_{a}\left(1-\omega_{b}\right) \theta_{b}\left(1-\omega_{a}\right)\left[\delta_{a}\left(r+\gamma_{a}\right)+\beta_{a} \theta_{a}\right]}{\mu_{a}\left(r+\gamma_{a}\right)^{2}\left(r+\gamma_{b}\right)}-\frac{\left(1-\omega_{b}\right)^{2}\left[\delta_{b}\left(r+\gamma_{b}\right)+\beta_{b} \theta_{b}\right]^{2}}{2 \mu_{b} r\left(r+\gamma_{b}\right)^{2}},
\end{aligned}
$$

$$
\begin{aligned}
V_{s}^{*}= & \frac{\omega_{a} M+\omega_{b} N}{r}+\left[\frac{\lambda \gamma_{a}\left(1-\omega_{b}\right) \theta_{b}}{\left(r+\gamma_{a}\right)\left(r+\gamma_{b}\right)}-\frac{\omega_{a} \theta_{a}}{r+\gamma_{a}}\right] R_{a}-\frac{\omega_{b} \theta_{b}}{r+\gamma_{b}} R_{b} \\
& -\frac{\left\{\omega_{a}\left[\varepsilon_{a}\left(r+\gamma_{a}\right)+\alpha_{a} \theta_{a}\right]+\left(\eta r \alpha_{a} \omega_{b} \theta_{b} /\left(r+\gamma_{b}\right)\right)\right\}^{2}}{2 \mu_{s} r\left(r+\gamma_{a}\right)^{2}}-\frac{\omega_{2}^{2}\left[\varepsilon_{b}\left(r+\gamma_{b}\right)+\alpha_{b} \theta_{b}\right]^{2}}{2 \mu_{s} r\left(r+\gamma_{b}\right)^{2}} \\
& -\frac{\left(1-\omega_{a}\right)\left[\delta_{a}\left(r+\gamma_{a}\right)+\beta_{a} \theta_{a}\right]\left\{\omega_{a}\left[\delta_{a}\left(r+\gamma_{a}\right)+\beta_{a} \theta_{a}\right]+\left(\eta r \beta_{a} \omega_{b} \theta_{b} /\left(r+\gamma_{b}\right)\right)\right\}}{\mu_{a} r\left(r+\gamma_{a}\right)^{2}}-\frac{\omega_{b}\left(1-\omega_{b}\right)\left[\delta_{b}\left(r+\gamma_{b}\right)+\beta_{b} \theta_{b}\right]^{2}}{\mu_{b} r\left(r+\gamma_{b}\right)^{2}} .
\end{aligned}
$$


At this time, the total environmental pollution losses in the region are

$$
\begin{aligned}
V^{*}\left(R_{a}, R_{b}\right)= & V_{a}^{*}+V_{b}^{*}+V_{s}^{*} \\
= & \frac{M+N}{r}+\left[\frac{\lambda \gamma_{a}\left(1-\omega_{b}\right) \theta_{b}}{\left(r+\gamma_{a}\right)\left(r+\gamma_{b}\right)}-\frac{\omega_{a} \theta_{a}}{r+\gamma_{a}}\right] R_{a}-\frac{\theta_{b}}{r+\gamma_{b}} R_{b} \\
& -\frac{\left\{\omega_{a}\left[\varepsilon_{a}\left(r+\gamma_{a}\right)+\alpha_{a} \theta_{a}\right]+\left(\eta r \alpha_{a} \omega_{b} \theta_{b} /\left(r+\gamma_{b}\right)\right)\right\}\left\{\left(2-\omega_{a}\right)\left[\varepsilon_{a}\left(r+\gamma_{a}\right)+\alpha_{a} \theta_{a}\right]+\left(\eta r \alpha_{a} \omega_{b} \theta_{b} /\left(r+\gamma_{b}\right)\right)\right\}}{2 \mu_{s} r\left(r+\gamma_{a}\right)^{2}} \\
& -\frac{\omega_{b}\left(2-\omega_{b}\right)\left[\varepsilon_{b}\left(r+\gamma_{b}\right)+\alpha_{b} \theta_{b}\right]^{2}}{2 \mu_{s} r\left(r+\gamma_{b}\right)^{2}} \\
& -\frac{\left(1-\omega_{a}\right)\left[\delta_{a}\left(r+\gamma_{a}\right)+\beta_{a} \theta_{a}\right]\left\{\left(1+\omega_{a}\right)\left[\delta_{a}\left(r+\gamma_{a}\right)+\beta_{a} \theta_{a}\right]+\left(2 \eta r \beta_{a} \omega_{b} \theta_{b} /\left(r+\gamma_{b}\right)\right)\right\}}{2 \mu_{a} r\left(r+\gamma_{a}\right)^{2}} \\
& -\frac{\left(1-\omega_{b}\right)^{2}\left[\delta_{b}\left(r+\gamma_{b}\right)+\beta_{b} \theta_{b}\right]^{2}}{2 \mu_{b} r\left(r+\gamma_{b}\right)^{2}} .
\end{aligned}
$$

Find the partial derivatives for equations (30)-(32) and bring the results into equations (14)-(17). It is easy to get equations (7)-(10).
Under the equilibrium of the Nash game model, the environmental pollution control level of Local-gov $\mathrm{A}$ is

$$
\left\{\begin{array}{l}
R_{a}^{*}(t)=\frac{1}{\gamma_{a}}\left[\frac{\omega_{a} \alpha_{a}}{\mu_{s}}\left(\varepsilon_{a}+\frac{\alpha_{a} \theta_{a}}{r+\gamma_{a}}\right)+\frac{\left(1-\omega_{a}\right) \beta_{a}}{\mu_{s}}\left(\delta_{a}+\frac{\beta_{a} \theta_{a}}{r+\gamma_{b}}\right)-e^{-\gamma_{a} t+D_{a}}\right], \\
R_{a}^{*}(0)=R_{a} .
\end{array}\right.
$$
is

The environmental pollution control level of Local-gov B

$$
\left\{\begin{array}{l}
R_{b}^{*}(t)=\frac{\eta}{\gamma_{b}}\left[\frac{\omega_{a} \alpha_{a}}{\mu_{s}}\left(\varepsilon_{a}+\frac{\alpha_{a} \theta_{a}}{r+\gamma_{a}}\right)+\frac{\left(1-\omega_{a}\right) \beta_{a}}{\mu_{a}}\left(\delta_{a}+\frac{\beta_{a} \theta_{a}}{r+\gamma_{a}}\right)-e^{-\gamma_{a} t+D_{a}}\right] \\
+\frac{1}{\gamma_{b}}\left[\frac{\omega_{b} \alpha_{b}}{\mu_{s}}\left(\varepsilon_{b}+\frac{\alpha_{b} \theta_{b}}{r+\gamma_{b}}\right)+\frac{\left(1-\omega_{b}\right) \beta_{b}}{\mu_{b}}\left(\delta_{b}+\frac{\beta_{b} \theta_{b}}{r+\gamma_{b}}\right)-e^{-\gamma_{b} t+D_{b}}\right], \\
R_{b}^{*}(0)=R_{b}, \\
e^{D_{a}}=\frac{\omega_{a} \alpha_{a}}{\mu_{s}}\left(\varepsilon_{a}+\frac{\alpha_{a} \theta_{a}}{r+\gamma_{a}}\right)+\frac{\left(1-\omega_{a}\right) \beta_{a}}{\mu_{a}}\left(\delta_{a}+\frac{\beta_{a} \theta_{a}}{r+\gamma_{a}}\right)-\gamma_{a} R_{a},
\end{array}\right.
$$

where $D_{a}, D_{b}$ are arbitrary constants.

Equations (7) and (8) indicate that under the Nash game model, the effort level of the local government $E_{a}, E_{b}$ are negatively correlated with the cost coefficient $\mu$, the attenuation coefficient of environmental pollution control level $\gamma$, the discount rate $r$, and the share ratio of environmental 
pollution losses $\omega$; the effort level of the local government is positively related to the impact coefficient of local government effort on pollution losses $\delta$, the impact coefficient on environmental pollution control level $\beta$, and the impact coefficient of environmental pollution control level on pollution losses $\theta$. In other words, local governments should raise the environmental pollution control level from the aspects of environmental pollution control level, impact capacity, executive ability, and so on.

Equations (9) and (10) indicate that under the Nash game model, the Cent-gov's optimal effort levels $E_{a s}, E_{b s}$ are negatively correlated with the cost coefficient $\mu_{s}$, the attenuation coefficient of the environmental pollution control $\gamma$, and the discount rate $r$; the Cent-gov's optimal effort levels $E_{a s}, E_{b s}$ are positively correlated with the distribution ratio of environmental pollution losses $\omega$, the influence coefficient of the Cent-gov's efforts on environmental pollution losses $\varepsilon$, the impact coefficient of environmental pollution control level on pollution losses $\theta$, and the influence coefficient of the Cent-gov's effort on the environmental pollution control level $\alpha$. The Cent-gov should comprehensively consider the local government's environmental pollution control issues in terms of environmental pollution control level, impact capability, and executive ability to make decisions.

4.2. The Stackelberg Game Model. In order to improve the efficiency of resource allocation, the Cent-gov has made policy guidance through special fiscal expenditure, that is, the Cent-gov has undertaken a certain proportion of environmental pollution control costs for the local government, the funding ratios are $\lambda_{a}(t)$ and $\lambda_{b}(t)$, the Cent-gov first determines the funding ratios for Local-gov $A$ and Local-gov B, then the local governments select the appropriate effort level after observing the actions of the Cent-gov. The Cent-gov is the leader, the local governments are the followers, and the three parties launch the Stackelberg game.

The objective function of Local-gov A can be expressed as

$$
T_{a}=\int_{0}^{\infty} e^{-r t}\left\{\left(1-\omega_{a}\right)\left(M-\varepsilon_{a} E_{a s}-\delta_{a} E_{a}-\theta_{a} R_{a}\right)+\frac{\mu_{a}}{2}\left(1-\lambda_{a}\right) E_{a}^{2}\right\} \mathrm{d} t
$$

The objective function of Local-gov B can be expressed as $T_{b}=\int_{0}^{\infty} e^{-r t}\left\{\left(1-\omega_{b}\right)\left(N-\varepsilon_{b} E_{b s}-\delta_{b} E_{b}-\theta_{b} R_{b}\right)+\frac{\mu_{b}}{2}\left(1-\lambda_{b}\right) E_{b}^{2}\right\} \mathrm{d} t$.
The objective function of Cent-gov can be expressed as

$$
T_{s}=\int_{0}^{\infty} e^{-r t}\left\{\begin{array}{c}
\omega_{a}\left(M-\varepsilon_{a} E_{a s}-\delta_{a} E_{a}-\theta_{a} R_{a}\right)+\omega_{b}\left(N-\varepsilon_{b} E_{b s}-\delta_{b} E_{b}-\theta_{b} R_{b}\right) \\
+\frac{\mu_{s}}{2}\left(E_{a s}^{2}+E_{b s}^{2}\right)+\frac{1}{2}\left(\mu_{a} \lambda_{a} E_{a}^{2}+\mu_{b} \lambda_{b} E_{b}^{2}\right)
\end{array}\right\} \mathrm{d} t .
$$

Proposition 2. Under the Stackelberg game model, the static feedback equilibrium strategies of Local-gov A, Local-gov B, and Cent-gov are

$$
\begin{aligned}
E_{a}^{* *} & =\frac{1+\omega_{a}}{2 \mu_{a}}\left(\delta_{a}+\frac{\beta_{a} \theta_{a}}{r+\gamma_{a}}\right)+\frac{\eta r \beta_{a} \omega_{b} \theta_{b}}{\mu_{a}\left(r+\gamma_{a}\right)\left(r+\gamma_{b}\right)}, \\
E_{b}^{* *} & =\frac{1+\omega_{b}}{2 \mu_{b}}\left(\delta_{b}+\frac{\beta_{b} \theta_{b}}{r+\gamma_{b}}\right), \\
E_{a s}^{* *} & =\frac{\omega_{a}}{\mu_{s}}\left(\varepsilon_{a}+\frac{\alpha_{a} \theta_{a}}{r+\gamma_{a}}\right)+\frac{\eta r \alpha_{a} \omega_{b} \theta_{b}}{\mu_{s}\left(r+\gamma_{a}\right)\left(r+\gamma_{b}\right)}, \\
E_{b s}^{* *} & =\frac{\omega_{b}}{\mu_{s}}\left(\varepsilon_{b}+\frac{\alpha_{b} \theta_{b}}{r+\gamma_{b}}\right),
\end{aligned}
$$




$$
\begin{aligned}
& \lambda_{a}^{* *}= \begin{cases}0, & \left(0 \leq \omega_{a} \leq \frac{1}{3}\right), \\
\frac{\left(3 \omega_{a}-1\right)\left[\delta_{a}\left(r+\gamma_{a}\right)+\beta_{a} \theta_{a}\right]+\left(2 \eta r \beta_{a} \omega_{b} \theta_{b} /\left(r+\gamma_{b}\right)\right)}{\left(\omega_{a}+1\right)\left[\delta_{a}\left(r+\gamma_{a}\right)+\beta_{a} \theta_{a}\right]+\left(2 \eta r \beta_{a} \omega_{b} \theta_{b} /\left(r+\gamma_{b}\right)\right)} & \left(\frac{1}{3}<\omega_{a} \leq 1\right),\end{cases} \\
& \lambda_{b}^{* *}= \begin{cases}0, & \left(0 \leq \omega_{b} \leq \frac{1}{3}\right), \\
\frac{3 \omega_{b}-1}{\omega_{b}+1}, & \left(\frac{1}{3}<\omega_{b} \leq 1\right) .\end{cases}
\end{aligned}
$$

Proof. In order to obtain the Stackelberg equilibrium, the inverse induction method is used. Firstly, this paper assumes that there is a continuous bounded differential income function $V_{i}\left(R_{a}, R_{b}\right) i \in(a, b, s)$. The following HJB equations are satisfied for all $R_{a} \geq 0, R_{b} \geq 0$ :

$$
\begin{gathered}
r \cdot V_{a}\left(R_{a}, R_{b}\right)=\min _{E_{a} \geq 0}\left\{\begin{array}{c}
\left(1-\omega_{a}\right)\left(M-\varepsilon_{a} E_{a s}-\delta_{a} E_{a}-\theta_{a} R_{a}\right)+\frac{\mu_{a}}{2}\left(1-\lambda_{a}\right) E_{a}^{2} \\
-\left(\frac{\partial V_{a}}{\partial R_{a}}+\eta \frac{\partial V_{a}}{\partial R_{b}}\right)\left(\alpha_{a} E_{a s}+\beta_{a} E_{a}-\gamma_{a} R_{a}\right)-\frac{\partial V_{a}}{\partial R_{b}}\left(\alpha_{b} E_{b s}+\beta_{b} E_{b}-\gamma_{b} R_{b}\right)
\end{array}\right\}, \\
r \cdot V_{b}\left(R_{a}, R_{b}\right)=\min _{E_{b} \geq 0}\left\{\begin{array}{c}
\left(1-\omega_{b}\right)\left(N-\varepsilon_{b} E_{a s}-\delta_{b} E_{a}-\theta_{b} R_{b}\right)+\frac{\mu_{b}}{2}\left(1-\lambda_{b}\right) E_{b}^{2} \\
-\left(\frac{\partial V_{b}}{\partial R_{a}}+\eta \frac{\partial V_{b}}{\partial R_{b}}\right)\left(\alpha_{a} E_{a s}+\beta_{a} E_{a}-\gamma_{a} R_{a}\right)-\frac{\partial V_{b}}{\partial R_{b}}\left(\alpha_{b} E_{b s}+\beta_{b} E_{b}-\gamma_{b} R_{b}\right)
\end{array}\right\} .
\end{gathered}
$$

Find the first-order partial derivatives of the effort level $E_{a}, E_{b}$ from the expressions in the right-hand brace of equations (45) and (46), making them equal to zero, we can get

$$
\begin{aligned}
& E_{a}=\frac{\left(1-\omega_{a}\right) \delta_{a}+\beta_{a}\left(\left(\partial V_{a} / \partial R_{a}\right)+\eta\left(\partial V_{a} / \partial R_{b}\right)\right)}{\mu_{a}\left(1-\lambda_{a}\right)} \\
& E_{b}=\frac{\left(1-\omega_{b}\right) \delta_{b}+\beta_{b}\left(\partial V_{b} / \partial R_{b}\right)}{\mu_{b}\left(1-\lambda_{b}\right)}
\end{aligned}
$$

The Cent-gov rationally predicts that Local-gov $A$ and Local-gov B will choose their efforts functions $E_{a}, E_{b}$ according to the above formulas. Therefore, the Cent-gov should determine its own efforts strategy and funding ratio according to the rational response of local governments. The Cent-gov's HJB equation is

$$
r \cdot V_{s}\left(R_{a}, R_{b}\right)=\min _{E_{a s} \geq 0, E_{b s} \geq 0}\left\{\begin{array}{c}
\omega_{a}\left(M-\varepsilon_{a} E_{a s}-\delta_{a} E_{a}-\theta_{a} R_{a}\right)+\omega_{b}\left(N-\varepsilon_{b} E_{b s}-\delta_{b} E_{b}-\theta_{b} R_{b}\right) \\
+\frac{\lambda_{a} \mu_{a}}{2} E_{a}^{2}+\frac{\lambda_{b} \mu_{b}}{2} E_{b}^{2}+\frac{\mu_{s}}{2}\left(E_{a s}^{2}+E_{b s}^{2}\right) \\
-\left(\frac{\partial V_{s}}{\partial R_{a}}+\eta \frac{\partial V_{s}}{\partial R_{b}}\right)\left(\alpha_{a} E_{a s}+\beta_{a} E_{a}-\gamma_{a} R_{a}\right)-\frac{\partial V_{s}}{\partial R_{b}}\left(\alpha_{b} E_{b s}+\beta_{b} E_{b}-\gamma_{b} R_{b}\right)
\end{array}\right\} .
$$

Substitute formulas (47) and (48) into formula (49), and solve the right part of formula (49). Solving method is as follows: find the first-order partial derivative of the formula in parentheses on the right end of the formula (49) with respect to $E_{a s}, E_{b s}, \eta_{a}, \eta_{b}$ and then make the first-order partial derivative equal to zero, and it is easy to obtain: 


$$
\begin{aligned}
E_{a s}= & \frac{\omega_{s} \varepsilon_{a}+\alpha_{a}\left(\left(\partial V_{s} / \partial R_{a}\right)+\eta\left(\partial V_{s} / \partial R_{b}\right)\right)}{\mu_{s}}, \\
E_{b s}= & \frac{\omega_{b} \varepsilon_{b}+\alpha_{b}\left(\partial V_{s} / \partial R_{b}\right)}{\mu_{b}}, \\
\lambda_{a}= & \frac{\left(3 \omega_{a}-1\right) \delta_{a}+\beta_{a}\left[2\left(\left(\partial V_{s} / \partial R_{a}\right)+\eta\left(\partial V_{s} / \partial R_{b}\right)\right)-\left(\left(\partial V_{a} / \partial R_{a}\right)+\eta\left(\partial V_{a} / \partial R_{b}\right)\right)\right]}{\left(\omega_{a}+1\right) \delta_{a}+\beta_{a}\left[2\left(\left(\partial V_{s} / \partial R_{a}\right)+\eta\left(\partial V_{s} / \partial R_{b}\right)\right)+\left(\left(\partial V_{a} / \partial R_{a}\right)+\eta\left(\partial V_{a} / \partial R_{b}\right)\right)\right]}, \\
\lambda_{b}= & \frac{\left(3 \omega_{b}-1\right) \delta_{b}+\beta_{b}\left(2\left(\partial V_{s} / \partial R_{b}\right)-\left(\partial V_{b} / \partial R_{b}\right)\right)}{\left(\omega_{b}+1\right) \delta_{b}+\beta_{b}\left(2\left(\partial V_{s} / \partial R_{b}\right)+\left(\partial V_{b} / \partial R_{b}\right)\right)} .
\end{aligned}
$$

Substituting equations (47), (48), and (50)-(53) into equations (45), (46), and (49), by simplifying, we can get

$$
\begin{aligned}
& r \cdot V_{a}=\left[\gamma_{a}\left(\frac{\partial V_{a}}{\partial R_{a}}+\eta \frac{\partial V_{a}}{\partial R_{b}}\right)-\left(1-\omega_{a}\right) \theta_{a}\right] R_{a}+\gamma_{b} \frac{\partial V_{a}}{\partial R_{b}} R_{b} \\
& -\frac{\left[\omega_{a} \varepsilon_{a}+\alpha_{a}\left(\left(\partial V_{s} / \partial R_{a}\right)+\eta\left(\partial V_{s} / \partial R_{b}\right)\right)\right]\left[\left(1-\omega_{a}\right) \varepsilon_{a}+\alpha_{a}\left(\left(\partial V_{a} / \partial R_{a}\right)+\eta\left(\partial V_{a} / \partial R_{b}\right)\right)\right]}{\mu_{s}}-\frac{\alpha_{b}\left(\partial V_{a} / \partial R_{b}\right)\left(\omega_{b} \varepsilon_{b}+\alpha_{b}\left(\partial V_{s} / \partial R_{b}\right)\right)}{\mu_{s}} \\
& -\frac{\left[\left(1-\omega_{a}\right) \delta_{a}+\beta_{a}\left(\left(\partial V_{a} / \partial R_{a}\right)+\eta\left(\partial V_{a} / \partial R_{b}\right)\right)\right]\left\{\left(1+\omega_{a}\right) \delta_{a}+\beta_{a}\left[2\left(\left(\partial V_{s} / \partial R_{a}\right)+\eta\left(\partial V_{s} / \partial R_{b}\right)\right)+\left(\left(\partial V_{a} / \partial R_{a}\right)+\eta\left(\partial V_{a} / \partial R_{b}\right)\right)\right]\right\}}{4 \mu_{a}} \\
& -\frac{\beta_{b}\left(\partial V_{a} / \partial R_{b}\right)\left[\left(1-\omega_{b}\right) \delta_{b}+\beta_{b}\left(\partial V_{s} / \partial R_{b}\right)\right]}{2 \mu_{b}}+\left(1-\omega_{a}\right) M \\
& r \cdot V_{b}=\gamma_{a}\left(\frac{\partial V_{b}}{\partial R_{a}}+\eta \frac{\partial V_{b}}{\partial R_{b}}\right) R_{a}+\left[\gamma_{b} \frac{\partial V_{b}}{\partial R_{b}}-\left(1-\omega_{b}\right) \theta_{b}\right] R_{b} \\
& -\frac{\left(\omega_{b} \varepsilon_{b}+\alpha_{b}\left(\partial V_{s} / \partial R_{b}\right)\right)\left[\left(1-\omega_{b}\right) \varepsilon_{b}+\alpha_{b}\left(\partial V_{b} / \partial R_{b}\right)\right]}{\mu_{s}} \\
& -\frac{\alpha_{a}\left(\left(\partial V_{b} / \partial R_{a}\right)+\eta\left(\partial V_{b} / \partial R_{b}\right)\right)\left[\omega_{a} \varepsilon_{a}+\alpha_{a}\left(\left(\partial V_{s} / \partial R_{a}\right)+\eta\left(\partial V_{s} / \partial R_{b}\right)\right)\right]}{\mu_{s}} \\
& -\frac{\beta_{a}\left(\left(\partial V_{b} / \partial R_{a}\right)+\eta\left(\partial V_{b} / \partial R_{b}\right)\right)\left\{\left(1+\omega_{a}\right) \delta_{a}+\beta_{a}\left[2\left(\left(\partial V_{s} / \partial R_{a}\right)+\eta\left(\partial V_{s} / \partial R_{b}\right)\right)+\left(\left(\partial V_{a} / \partial R_{a}\right)+\eta\left(\partial V_{a} / \partial R_{b}\right)\right)\right]\right\}}{2 \mu_{a}} \\
& -\frac{\left[\left(1-\omega_{b}\right) \delta_{b}+\beta_{b}\left(\partial V_{b} / \partial R_{b}\right)\right]\left[\left(1+\omega_{b}\right) \delta_{b}+\beta_{b}\left(2\left(\partial V_{s} / \partial R_{b}\right)+\left(\partial V_{b} / \partial R_{b}\right)\right)\right]}{4 \mu_{b}}+\left(1-\omega_{b}\right) N \\
& r \cdot V_{s}=\left[\gamma_{a}\left(\frac{\partial V_{s}}{\partial R_{a}}+\eta \frac{\partial V_{s}}{\partial R_{b}}\right)-\omega_{a} \theta_{a}\right] R_{a}+\left(\gamma_{b} \frac{\partial V_{s}}{\partial R_{b}}-\omega_{b} \theta_{b}\right) R_{b} \\
& -\frac{\left[\omega_{a} \varepsilon_{a}+\alpha_{a}\left(\left(\partial V_{s} / \partial R_{a}\right)+\eta\left(\partial V_{s} / \partial R_{b}\right)\right)\right]^{2}}{2 \mu_{s}}-\frac{\left(\omega_{b} \varepsilon_{b}+\alpha_{b}\left(\partial V_{s} / \partial R_{b}\right)\right)^{2}}{2 \mu_{s}} \\
& -\frac{\left[\omega_{a} \delta_{a}+\beta_{a}\left(\left(\partial V_{s} / \partial R_{a}\right)+\eta\left(\partial V_{s} / \partial R_{b}\right)\right)\right]\left[\omega_{a} \delta_{a}+\beta_{a}\left(\left(\partial V_{s} / \partial R_{a}\right)+\eta\left(\partial V_{s} / \partial R_{b}\right)+\left(\partial V_{a} / \partial R_{a}\right)+\eta\left(\partial V_{a} / \partial R_{b}\right)\right)\right]}{2 \mu_{a}} \\
& -\frac{\left[\left(1-\omega_{a}\right) \delta_{a}+\beta_{a}\left(\left(\partial V_{a} / \partial R_{a}\right)+\eta\left(\partial V_{a} / \partial R_{b}\right)\right)\right]^{2}}{8 \mu_{a}}-\frac{\left[\omega_{b} \delta_{b}+\beta_{b}\left(\left(\partial V_{s} / \partial R_{a}\right)+\left(\partial V_{s} / \partial R_{b}\right)\right)\right]\left(\delta_{b}+\beta_{b}\left(\partial V_{b} / \partial R_{b}\right)\right)}{\mu_{b}} \\
& -\frac{\left[\left(1-\omega_{b}\right) \delta_{b}+\beta_{b}\left(\partial V_{b} / \partial R_{b}\right)\right]^{2}}{8 \mu_{b}}+\omega_{a} M+\omega_{b} N
\end{aligned}
$$


From equations (54)-(56), it is known that the linear optimal functions about $R_{a}, R_{b}$ are the solutions of the HJB equation. Let

$$
\begin{aligned}
& V_{a}\left(R_{a}, R_{b}\right)=p_{a} R_{a}+q_{a} R_{b}+u_{a}, \\
& V_{b}\left(R_{a}, R_{b}\right)=p_{b} R_{a}+q_{b} R_{b}+u_{b},
\end{aligned}
$$

$$
\begin{aligned}
r\left(p_{a} R_{a}+q_{a} R_{b}+u_{a}\right)= & {\left[\gamma_{a}\left(p_{a}+\eta q_{a}\right)-\left(1-\omega_{a}\right) \theta_{a}\right] R_{a}+\gamma_{b} q_{a} R_{b} } \\
& -\frac{\left[\omega_{a} \varepsilon_{a}+\alpha_{a}\left(p_{s}+\eta q_{s}\right)\right]\left[\left(1-\omega_{a}\right) \varepsilon_{a}+\alpha_{a}\left(p_{a}+\eta q_{a}\right)\right]}{\mu_{s}}-\frac{\alpha_{b} q_{a}\left(\omega_{b} \varepsilon_{b}+\alpha_{b} q_{s}\right)}{\mu_{s}} \\
& -\frac{\left[\left(1-\omega_{a}\right) \delta_{a}+\beta_{a}\left(p_{a}+\eta q_{a}\right)\right]\left\{\left(1+\omega_{a}\right) \delta_{a}+\beta_{a}\left[2\left(p_{s}+\eta q_{s}\right)+\left(p_{a}+\eta q_{a}\right)\right]\right\}}{4 \mu_{a}} \\
& -\frac{\beta_{b} q_{a}\left[\left(1-\omega_{b}\right) \delta_{b}+\beta_{b} q_{b}\right]}{2 \mu_{b}}+\left(1-\omega_{a}\right) M,
\end{aligned}
$$

$$
\begin{aligned}
r\left(p_{b} R_{a}+q_{b} R_{b}+u_{b}\right)= & \gamma_{a}\left(p_{b}+\eta q_{b}\right) R_{a}+\left[\gamma_{b} q_{b}-\left(1-\omega_{b}\right) \theta_{b}\right] R_{b} \\
& -\frac{\left(\omega_{b} \varepsilon_{b}+\alpha_{b} q_{s}\right)\left[\left(1-\omega_{b}\right) \varepsilon_{b}+\alpha_{b} q_{b}\right]}{\mu_{s}}-\frac{\alpha_{a}\left(p_{b}+\eta q_{b}\right)\left[\omega_{a} \varepsilon_{a}+\alpha_{a}\left(p_{s}+\eta q_{s}\right)\right]}{\mu_{s}} \\
& -\frac{\beta_{a}\left(p_{b}+\eta q_{b}\right)\left\{\left(1+\omega_{a}\right) \delta_{a}+\beta_{a}\left[2\left(p_{s}+\eta q_{s}\right)+\left(p_{a}+\eta q_{a}\right)\right]\right\}}{2 \mu_{a}} \\
& -\frac{\left[\left(1-\omega_{b}\right) \delta_{b}+\beta_{b} q_{b}\right]\left[\left(1+\omega_{b}\right) \delta_{b}+\beta_{b}\left(2 q_{s}+q_{b}\right)\right]}{4 \mu_{b}}+\left(1-\omega_{b}\right) N, \\
r\left(p_{s} R_{a}+q_{s} R_{b}+u_{s}\right)= & {\left[\gamma_{a}\left(p_{s}+\eta q_{s}\right)-\omega_{a} \theta_{a}\right] R_{a}+\left(\gamma_{b} q_{s}-\omega_{b} \theta_{b}\right) R_{b} } \\
& -\frac{\left[\omega_{a} \varepsilon_{a}+\alpha_{a}\left(p_{s}+\eta q_{s}\right)\right]^{2}}{2 \mu_{s}}-\frac{\left(\omega_{b} \varepsilon_{b}+\alpha_{b} q_{s}\right)^{2}}{2 \mu_{s}}-\frac{\left[\omega_{a} \delta_{a}+\beta_{a}\left(p_{s}+\eta q_{s}\right)\right]\left[\omega_{a} \delta_{a}+\beta_{a}\left(p_{s}+\eta q_{s}+p_{a}+\eta q_{a}\right)\right]}{2 \mu_{a}} \\
& -\frac{\left[\left(1-\omega_{a}\right) \delta_{a}+\beta_{a}\left(p_{s}+\eta q_{s}\right)\right]^{2}}{8 \mu_{a}}-\frac{\left[\omega_{b} \delta_{b}+\beta_{b} q_{s}\right]\left[\delta_{b}+\beta_{b}\left(q_{b}+q_{s}\right)\right]}{2 \mu_{b}} \\
& -\frac{\left[\left(1-\omega_{b}\right) \delta_{b}+\beta_{b}\left(p_{a}+\eta q_{a}\right)\right]^{2}}{8 \mu_{b}}+\omega_{a} M+\omega_{b} N .
\end{aligned}
$$

If equations (60)-(62) satisfy all $R_{a} \geq 0, R_{b} \geq 0$, it is easy to get

$$
\begin{aligned}
p_{a}= & -\frac{\left(1-\omega_{a}\right) \theta_{a}}{r+\gamma_{a}}, \\
p_{b}= & 0, \\
u_{a}= & -\frac{\left(1-\omega_{a}\right)\left[\varepsilon_{a}\left(r+\gamma_{a}\right)+\alpha_{a} \theta_{a}\right]\left\{\omega_{a}\left[\varepsilon_{a}\left(r+\gamma_{a}\right)+\alpha_{a} \theta_{a}\right]+\left(\eta r \alpha_{a} \omega_{b} \theta_{b} /\left(r+\gamma_{b}\right)\right)\right\}}{\mu_{s} r\left(r+\gamma_{a}\right)^{2}} \\
& -\frac{\left(1-\omega_{a}\right)\left[\delta_{a}\left(r+\gamma_{a}\right)+\beta_{a} \theta_{a}\right]\left\{\left(1+\omega_{a}\right)\left[\delta_{a}\left(r+\gamma_{a}\right)+\beta_{a} \theta_{a}\right]+\left(2 \eta r \beta_{a} \omega_{b} \theta_{b} /\left(r+\gamma_{b}\right)\right)\right\}}{4 \mu_{a} r\left(r+\gamma_{a}\right)^{2}}+\frac{\left(1-\omega_{a}\right) M}{r},
\end{aligned}
$$




$$
\begin{aligned}
p_{b}= & \frac{\lambda \gamma_{a}\left(1-\omega_{b}\right) \theta_{b}}{\left(r+\gamma_{a}\right)\left(r+\gamma_{b}\right)}, \\
q_{b}= & -\frac{\left(1-\omega_{b}\right) \theta_{b}}{r+\gamma_{b}}, \\
u_{b}= & -\frac{\eta \alpha_{a}\left(1-\omega_{b}\right) \theta_{b}\left\{\omega_{a}\left[\varepsilon_{a}\left(r+\gamma_{a}\right)+\alpha_{a} \theta_{a}\right]+\left(\eta r \alpha_{a} \omega_{b} \theta_{b} /\left(r+\gamma_{b}\right)\right)\right\}}{\mu_{s}\left(r+\gamma_{a}\right)^{2}\left(r+\gamma_{b}\right)}-\frac{\omega_{b}\left(1-\omega_{b}\right)\left[\varepsilon_{b}\left(r+\gamma_{b}\right)+\alpha_{b} \theta_{b}\right]^{2}}{\mu_{s} r\left(r+\gamma_{b}\right)^{2}} \\
& +\frac{\left(1-\omega_{b}\right) N}{r}-\frac{\eta \beta_{a}\left(1-\omega_{b}\right) \theta_{b}\left\{\left(1+\omega_{a}\right)\left[\delta_{a}\left(r+\gamma_{a}\right)+\beta_{a} \theta_{a}\right]+\left(2 \eta r \alpha_{a} \omega_{b} \theta_{b} /\left(r+\gamma_{b}\right)\right)\right\}}{2 \mu_{a}\left(r+\gamma_{a}\right)^{2}\left(r+\gamma_{b}\right)} \\
& -\frac{\left(1-\omega_{b}\right)\left(1+\omega_{b}\right)\left[\delta_{b}\left(r+\gamma_{b}\right)+\beta_{b} \theta_{b}\right]^{2}}{4 \mu_{b} r\left(r+\gamma_{b}\right)^{2}}, \\
p_{s}= & \frac{\lambda \gamma_{a}\left(1-\omega_{b}\right) \theta_{b}}{\left(r+\gamma_{a}\right)\left(r+\gamma_{b}\right)}-\frac{\omega_{a} \theta_{a}}{r+\gamma_{a}}, \\
q_{s}= & -\frac{\omega_{2} \theta_{b}}{r+\gamma_{b}}, \\
u_{s}= & -\frac{\left\{\omega_{a}\left[\varepsilon_{a}\left(r+\gamma_{a}\right)+\alpha_{a} \theta_{a}\right]+\left(\eta r \alpha_{a} \omega_{b} \theta_{b} /\left(r+\gamma_{b}\right)\right)\right\}^{2}}{2 \mu_{s} r\left(r+\gamma_{a}\right)^{2}}-\frac{\omega_{b}^{2}\left[\varepsilon_{b}\left(r+\gamma_{b}\right)+\alpha_{b} \theta_{b}\right]^{2}}{2 \mu_{s} r\left(r+\gamma_{b}\right)^{2}} \\
& -\frac{\left\{\left(1+\omega_{a}\right)\left[\delta_{a}\left(r+\gamma_{a}\right)+\beta_{a} \theta_{a}\right]+\left(2 \eta r \beta_{a} \omega_{b} \theta_{b} /\left(r+\gamma_{b}\right)\right)\right\}^{2}}{8 \mu_{a} r\left(r+\gamma_{a}\right)^{2}}-\frac{\left(1+\omega_{b}\right)^{2}\left[\delta_{b}\left(r+\gamma_{b}\right)+\beta_{b} \theta_{b}\right]^{2}}{8 \mu_{b} r\left(r+\gamma_{b}\right)^{2}}+\frac{\omega_{a} M+\omega_{b} N}{r} .
\end{aligned}
$$

Substituting equations (63)-(65) into equations (57)-(59), the minimum environmental pollution losses functions of Local-gov A, Local-gov B, and Cent-gov can be obtained, they are

$$
\begin{aligned}
V_{a}^{* *}= & \frac{\left(1-\omega_{a}\right) M}{r}-\frac{\left(1-\omega_{a}\right) \theta_{a}}{r+\gamma_{a}} R_{a}-\frac{\left(1-\omega_{a}\right)\left[\varepsilon_{a}\left(r+\gamma_{a}\right)+\alpha_{a} \theta_{a}\right]\left\{\omega_{a}\left[\varepsilon_{a}\left(r+\gamma_{a}\right)+\alpha_{a} \theta_{a}\right]+\left(\eta r \alpha_{a} \omega_{b} \theta_{b} /\left(r+\gamma_{b}\right)\right)\right\}}{\mu_{s} r\left(r+\gamma_{a}\right)^{2}} \\
& -\frac{\left(1-\omega_{a}\right)\left[\delta_{a}\left(r+\gamma_{a}\right)+\beta_{a} \theta_{a}\right]\left\{\left(1+\omega_{a}\right)\left[\delta_{a}\left(r+\gamma_{a}\right)+\beta_{a} \theta_{a}\right]+\left(2 \eta r \beta_{a} \omega_{b} \theta_{b} /\left(r+\gamma_{b}\right)\right)\right\}}{4 \mu_{a} r\left(r+\gamma_{a}\right)^{2}}, \\
V_{b}^{* *}= & \frac{\left(1-\omega_{b}\right) N}{r}+\frac{\lambda \gamma_{a}\left(1-\omega_{b}\right) \theta_{b}}{\left(r+\gamma_{a}\right)\left(r+\gamma_{b}\right)} R_{a}-\frac{\left(1-\omega_{b}\right) \theta_{b}}{r+\gamma_{b}} R_{b}-\frac{\eta \alpha_{a}\left(1-\omega_{b}\right) \theta_{b}\left\{\omega_{a}\left[\varepsilon_{a}\left(r+\gamma_{a}\right)+\alpha_{a} \theta_{a}\right]+\left(\eta r \alpha_{a} \omega_{b} \theta_{b} /\left(r+\gamma_{b}\right)\right)\right\}}{\mu_{s}\left(r+\gamma_{a}\right)^{2}\left(r+\gamma_{b}\right)} \\
& -\frac{\omega_{b}\left(1-\omega_{b}\right)\left[\varepsilon_{b}\left(r+\gamma_{b}\right)+\alpha_{b} \theta_{b}\right]^{2}}{\mu_{s} r\left(r+\gamma_{b}\right)^{2}}-\frac{\eta \beta_{a}\left(1-\omega_{b}\right) \theta_{b}\left\{\left(1+\omega_{a}\right)\left[\delta_{a}\left(r+\gamma_{a}\right)+\beta_{a} \theta_{a}\right]+\left(2 \eta r \alpha_{a} \omega_{b} \theta_{b} /\left(r+\gamma_{b}\right)\right)\right\}}{2 \mu_{a}\left(r+\gamma_{a}\right)^{2}\left(r+\gamma_{b}\right)} \\
& -\frac{\left(1-\omega_{b}\right)\left(1+\omega_{b}\right)\left[\delta_{b}\left(r+\gamma_{b}\right)+\beta_{b} \theta_{b}\right]^{2}}{4 \mu_{b} r\left(r+\gamma_{b}\right)^{2}},
\end{aligned}
$$

$$
\begin{aligned}
V_{s}^{* *}= & \frac{\omega_{a} M+\omega_{b} N}{r}+\left[\frac{\lambda \gamma_{a}\left(1-\omega_{b}\right) \theta_{b}}{\left(r+\gamma_{a}\right)\left(r+\gamma_{b}\right)}-\frac{\omega_{a} \theta_{a}}{r+\gamma_{a}}\right] R_{a}-\frac{\omega_{2} \theta_{b}}{r+\gamma_{b}} R_{b} \\
& -\frac{\left\{\omega_{a}\left[\varepsilon_{a}\left(r+\gamma_{a}\right)+\alpha_{a} \theta_{a}\right]+\left(\eta r \alpha_{a} \omega_{b} \theta_{b} /\left(r+\gamma_{b}\right)\right)\right\}^{2}}{2 \mu_{s} r\left(r+\gamma_{a}\right)^{2}}-\frac{\omega_{b}^{2}\left[\varepsilon_{b}\left(r+\gamma_{b}\right)+\alpha_{b} \theta_{b}\right]^{2}}{2 \mu_{s} r\left(r+\gamma_{b}\right)^{2}} \\
& -\frac{\left\{\left(1+\omega_{a}\right)\left[\delta_{a}\left(r+\gamma_{a}\right)+\beta_{a} \theta_{a}\right]+\left(2 \eta r \beta_{a} \omega_{b} \theta_{b} /\left(r+\gamma_{b}\right)\right)\right\}^{2}}{8 \mu_{a} r\left(r+\gamma_{a}\right)^{2}}-\frac{\left(1+\omega_{b}\right)^{2}\left[\delta_{b}\left(r+\gamma_{b}\right)+\beta_{b} \theta_{b}\right]^{2}}{8 \mu_{b} r\left(r+\gamma_{b}\right)^{2}} .
\end{aligned}
$$


At this time, the total environmental pollution regional losses are

$$
\begin{aligned}
V^{* *}\left(R_{a}, R_{b}\right)= & V_{a}^{* *}+V_{b}^{* *}+V_{s}^{* *} \\
= & \frac{M+N}{r}+\left[\frac{\lambda \gamma_{a} \theta_{b}}{\left(r+\gamma_{a}\right)\left(r+\gamma_{b}\right)}-\frac{\theta_{a}}{r+\gamma_{a}}\right] R_{a}-\frac{\theta_{b}}{r+\gamma_{b}} R_{b} \\
& -\frac{\left\{\omega_{a}\left[\varepsilon_{a}\left(r+\gamma_{a}\right)+\alpha_{a} \theta_{a}\right]+\left(\eta r \alpha_{a} \omega_{b} \theta_{b} /\left(r+\gamma_{b}\right)\right)\right\}\left\{\left(2-\omega_{a}\right)\left[\varepsilon_{a}\left(r+\gamma_{a}\right)+\alpha_{a} \theta_{a}\right]+\left(\eta r \alpha_{a}\left(2-\omega_{b}\right) \theta_{b} /\left(r+\gamma_{b}\right)\right)\right\}}{2 \mu_{s} r\left(r+\gamma_{a}\right)^{2}} \\
& -\frac{\omega_{b}\left(2-\omega_{b}\right)\left[\varepsilon_{b}\left(r+\gamma_{b}\right)+\alpha_{b} \theta_{b}\right]^{2}}{2 \mu_{s} r\left(r+\gamma_{b}\right)^{2}} \\
& -\frac{\left\{\left(1+\omega_{a}\right)\left[\delta_{a}\left(r+\gamma_{a}\right)+\beta_{a} \theta_{a}\right]+\left(2 \eta r \beta_{a} \omega_{b} \theta_{b} /\left(r+\gamma_{b}\right)\right)\right\}\left\{\left(3-\omega_{a}\right)\left[\delta_{a}\left(r+\gamma_{a}\right)+\beta_{a} \theta_{a}\right]+\left(2 \eta r \beta_{a}\left(2-\omega_{b}\right) \theta_{b} /\left(r+\gamma_{b}\right)\right)\right\}}{8 \mu_{a} r\left(r+\gamma_{a}\right)^{2}} \\
& -\frac{\left(3-\omega_{b}\right)\left(1+\omega_{b}\right)\left[\delta_{b}\left(r+\gamma_{b}\right)+\beta_{b} \theta_{b}\right]^{2}}{8 \mu_{b} r\left(r+\gamma_{b}\right)^{2}} .
\end{aligned}
$$

The partial derivatives of equations (65)-(68) are calculated and then substituted into the results of equations (47), (48), and (50)-(53) to obtain equations (39)-(44).
Under the Stackelberg game model, the environmental pollution control level of Local-gov A is

$$
\left\{\begin{array}{l}
R_{a}^{* *}(t)=\frac{1}{\gamma_{a}}\left[\frac{\alpha_{a}}{\mu_{s}}\left[\omega_{a}\left(\varepsilon_{a}+\frac{\alpha_{a} \theta_{a}}{r+\gamma_{a}}\right)+\frac{\eta r \alpha_{a} \omega_{b} \theta_{b}}{\left(r+\gamma_{a}\right)\left(r+\gamma_{b}\right)}\right]+\frac{\beta_{a}}{\mu_{a}}\left[\frac{1+\omega_{a}}{2}\left(\delta_{a}+\frac{\beta_{a} \theta_{a}}{r+\gamma_{b}}\right)+\frac{\eta r \beta_{a} \omega_{b} \theta_{b}}{\left(r+\gamma_{a}\right)\left(r+\gamma_{b}\right)}\right]-e^{-\gamma_{a} t+D_{a}}\right] \\
R_{a}^{* *}(0)=R_{a} .
\end{array}\right.
$$
is

The environmental pollution control level of Local-gov B

$$
\left\{\begin{array}{l}
R_{b}^{* *}(t)=\frac{\eta}{\gamma_{b}}\left\{\frac{\alpha_{a}}{\mu_{s}}\left[\omega_{a}\left(\varepsilon_{a}+\frac{\alpha_{a} \theta_{a}}{r+\gamma_{a}}\right)+\frac{\eta r \alpha_{a} \omega_{b} \theta_{b}}{\left(r+\gamma_{a}\right)\left(r+\gamma_{b}\right)}\right]+\frac{\beta_{a}}{\mu_{a}}\left[\frac{1+\omega_{a}}{2}\left(\delta_{a}+\frac{\beta_{a} \theta_{a}}{r+\gamma_{a}}\right)+\frac{\eta r \alpha_{a} \omega_{b} \theta_{b}}{\left(r+\gamma_{a}\right)\left(r+\gamma_{b}\right)}\right]-e^{-\gamma_{a} t+D_{a}}\right\} \\
+\frac{1}{\gamma_{b}}\left[\frac{\omega_{b} \alpha_{b}}{\mu_{s}}\left(\varepsilon_{b}+\frac{\alpha_{b} \theta_{b}}{r+\gamma_{b}}\right)+\frac{\left(1+\omega_{b}\right) \beta_{b}}{\mu_{b}}\left(\delta_{b}+\frac{\beta_{b} \theta_{b}}{r+\gamma_{b}}\right)-e^{-\gamma_{b} t+D_{b}}\right] \\
R_{b}^{* *}(0)=R_{b}, \\
e^{D_{a}} \&=\frac{\alpha_{a}}{\mu_{s}}\left[\omega_{a}\left(\varepsilon_{a}+\frac{\alpha_{a} \theta_{a}}{r+\gamma_{a}}\right)+\frac{\eta r \alpha_{a} \omega_{b} \theta_{b}}{\left(r+\gamma_{a}\right)\left(r+\gamma_{b}\right)}\right]+\frac{\beta_{a}}{\mu_{a}}\left[\frac{1+\omega_{a}}{2}\left(\delta_{a}+\frac{\beta_{a} \theta_{a}}{r+\gamma_{a}}\right)+\frac{\eta r \alpha_{a} \omega_{b} \theta_{b}}{\left(r+\gamma_{a}\right)\left(r+\gamma_{b}\right)}\right]-\gamma_{a} R_{a}
\end{array}\right.
$$


By comparing equation (7) and (39), equations (8) and (40), we know that under the Stackelberg game model, the effort levels of the local government $E_{a}, E_{b}$ are positively correlated with the distribution ratio of environmental pollution losses $\omega$, but the impact degree is less than the Nash game model, while other influencing factors and influence direction are consistent with the Nash game model.

By comparing equations (9) and (41), equations (10) and (42), it can be seen that under the Stackelberg game model, the effort level of Cent-gov and the related influencing factors are consistent with the Nash game model. In other words, the government must comprehensively consider its own environmental pollution governance level, impact ability, and execution ability to make decisions.

Equations (43) and (44) show that the funding ratio chosen by the Cent-gov depends on the sharing ratio of environmental pollution losses. When the Cent-gov bears less environmental pollution losses $(0 \leq \omega \leq(1 / 3))$, it will not consider sharing costs through the subsidy mechanism; when the Cent-gov bears more environmental pollution losses, it will consider improving the efficiency of environmental pollution control through special subsidy, which reflects the Cent-gov's "Economic person" characteristics.

4.3. The Cooperative Game Model. The Coase theorem shows that if transaction costs are low enough, the rational participants can achieve Pareto optimal allocation through voluntary bargaining. Under appropriate conditions, governments can achieve economies of scale and policy spillovers through cooperation. As the intergovernmental relations gradually develop in all directions, in many fields, and at a deeper level, the governments should build an intergovernmental coordination model. In order to further improve the efficiency of environmental pollution control, the Cent-gov cooperates with Local-gov A and Local-gov B to jointly determine the optimal effort strategy, improve the environmental pollution control level, and reduce environmental pollution losses.

Proposition 3. Under the situation of full communication and cooperation between the Cent-gov, Local-gov A, and Local-gov $B$, their optimal equilibrium strategies are

$$
E_{a}^{* * *}=\frac{1}{\mu_{a}}\left[\delta_{a}+\frac{\beta_{a} \theta_{a}}{r+\gamma_{a}}+\frac{\eta r \beta_{a} \theta_{b}}{\left(r+\gamma_{a}\right)\left(r+\gamma_{b}\right)}\right],
$$

$$
\begin{aligned}
E_{b}^{* * *} & =\frac{1}{\mu_{b}}\left(\delta_{b}+\frac{\beta_{b} \theta_{b}}{r+\gamma_{b}}\right), \\
E_{a s}^{* * *} & =\frac{1}{\mu_{s}}\left[\varepsilon_{a}+\frac{\alpha_{a} \theta_{a}}{r+\gamma_{a}}+\frac{\eta r \alpha_{a} \theta_{b}}{\mu_{s}\left(r+\gamma_{a}\right)\left(r+\gamma_{b}\right)}\right], \\
E_{b s}^{* * *} & =\frac{1}{\mu_{s}}\left(\varepsilon_{b}+\frac{\alpha_{b} \theta_{b}}{r+\gamma_{b}}\right) .
\end{aligned}
$$

Proof. When the relationship between the Cent-gov, Localgov $\mathrm{A}$, and Local-gov B is transformed into collaboration, all parties aim at minimizing environmental pollution losses regionally and jointly determine the optimal values of $E_{a}, E_{b}, E_{a s}$, and $E_{b s}$. The total environmental pollution losses can be expressed as

$$
T=\int_{0}^{\infty} e^{-r t}\left\{\begin{array}{c}
M-\varepsilon_{a} E_{a s}-\delta_{a} E_{a}-\theta_{a} R_{a}+N-\varepsilon_{b} E_{b s}-\delta_{b} E_{b}-\theta_{b} R_{b} \\
+\frac{1}{2}\left(\mu_{a} E_{a}^{2}+\mu_{b} E_{b}^{2}+\mu_{s} E_{a s}^{2}+\mu_{s} E_{b s}^{2}\right)
\end{array}\right\} \mathrm{d} t .
$$

Supposing there is a continuous bounded differential income function $V\left(R_{a}, R_{b}\right)$ that satisfies the HJB equation for all $R_{a} \geq 0, R_{b} \geq 0$,

$$
r \cdot V\left(R_{a}, R_{b}\right)=\min _{E_{a s} \geq 0 ; E_{a} \geq 0}\left\{\begin{array}{c}
M-\varepsilon_{a} E_{a s}-\delta_{a} E_{a}-\theta_{a} R_{a}+\frac{\mu_{s}}{2} E_{a s}^{2}+\frac{\mu_{a}}{2} E_{a}^{2}, \\
N-\varepsilon_{b} E_{b s}-\delta_{b} E_{b}-\theta_{b} R_{b}+\frac{\mu_{s}}{2} E_{b s}^{2}+\frac{\mu_{b}}{2} E_{b}^{2} \\
-\left(\frac{\partial V}{\partial L_{1}}+\eta \frac{\partial V}{\partial L_{2}}\right)\left(\alpha_{a} E_{a s}+\beta_{a} E_{a}-\gamma_{a} R_{a}\right)-\frac{\partial V}{\partial L_{2}}\left(\alpha_{b} E_{b s}+\beta_{b} E_{b}-\gamma_{b} R_{b}\right) .
\end{array}\right\}
$$


In equation (77), calculating the first-order partial derivatives of $E_{a s}, E_{b s}, E_{a}, E_{b}$ and making them equal to 0 , we can get

$$
\begin{aligned}
& E_{a}=\frac{\delta_{a}+\beta_{a}\left(\left(\partial V / \partial R_{a}\right)+\eta\left(\partial V / \partial R_{b}\right)\right)}{\mu_{a}}, \\
& E_{b}=\frac{\delta_{b}+\beta_{b}\left(\partial V / \partial R_{b}\right)}{\mu_{b}}
\end{aligned}
$$

$$
\begin{aligned}
& E_{a s}=\frac{\varepsilon_{a}+\alpha_{a}\left(\left(\partial V / \partial R_{a}\right)+\eta\left(\partial V / \partial R_{b}\right)\right)}{\mu_{s}}, \\
& E_{b s}=\frac{\varepsilon_{b}+\alpha_{b}\left(\partial V / \partial R_{b}\right)}{\mu_{b}} .
\end{aligned}
$$

Substituting the formulas (78)-(81) into formula (77) for simplification yields

$$
\begin{aligned}
r \cdot V= & {\left[\gamma_{a}\left(\frac{\partial V}{\partial R_{a}}+\eta \frac{\partial V}{\partial R_{b}}\right)-\theta_{a}\right] R_{a}+\left(\gamma_{b} \frac{\partial V}{\partial R_{b}}-\theta_{b}\right) R_{b}-\frac{\left[\varepsilon_{a}+\alpha_{a}\left(\left(\partial V_{s} / \partial R_{a}\right)+\eta\left(\partial V_{s} / \partial R_{b}\right)\right)\right]^{2}}{2 \mu_{s}} } \\
& -\frac{\left(\varepsilon_{b}+\alpha_{b}\left(\partial V_{s} / \partial R_{b}\right)\right)^{2}}{2 \mu_{s}}-\frac{\left[\delta_{a}+\beta_{a}\left(\left(\partial V / \partial R_{a}\right)+\eta\left(\partial V / \partial R_{b}\right)\right)\right]^{2}}{2 \mu_{a}}-\frac{\left(\delta_{b}+\beta_{b}\left(\partial V / \partial R_{b}\right)\right)^{2}}{2 \mu_{b}}+\omega_{a} M+\omega_{b} N .
\end{aligned}
$$

From equation (81), we can see that the linear optimal income functions about $R_{a}, R_{b}$ are the solutions of the HJB equation, let

$$
V\left(R_{a}, R_{b}\right)=p R_{a}+q R_{b}+u,
$$

$$
\begin{aligned}
r\left(p R_{a}+q R_{b}+u\right)= & {\left[\gamma_{a}(p+\eta q)-\theta_{a}\right] R_{a}+\left(\gamma_{b} q-\theta_{b}\right) R_{b}-\frac{\left[\varepsilon_{a}+\alpha_{a}(p+\eta q)\right]^{2}}{2 \mu_{s}}-\frac{\left(\varepsilon_{b}+\alpha_{b} q\right)^{2}}{2 \mu_{s}} } \\
& -\frac{\left[\delta_{a}+\beta_{a}(p+\eta q)\right]^{2}}{2 \mu_{a}}-\frac{\left(\delta_{b}+\beta_{b} q\right)^{2}}{2 \mu_{b}}+\omega_{a} M+\omega_{b} N .
\end{aligned}
$$

If formula (84) satisfies all $R_{a} \geq 0, R_{b} \geq 0$, we can get

$$
\begin{aligned}
p= & \frac{\lambda \gamma_{a} \theta_{b}}{\left(r+\gamma_{a}\right)\left(r+\gamma_{b}\right)}-\frac{\theta_{a}}{r+\gamma_{a}}, \\
q= & -\frac{\theta_{b}}{r+\gamma_{b}}, \\
u= & -\frac{\left[\varepsilon_{a}\left(r+\gamma_{a}\right)+\alpha_{a} \theta_{a}+\left(\eta r \alpha_{a} \theta_{b} /\left(r+\gamma_{b}\right)\right)\right]^{2}}{2 \mu_{s} r\left(r+\gamma_{a}\right)^{2}}-\frac{\left[\varepsilon_{b}\left(r+\gamma_{b}\right)+\alpha_{b} \theta_{b}\right]^{2}}{2 \mu_{s} r\left(r+\gamma_{b}\right)^{2}}-\frac{\left[\delta_{a}\left(r+\gamma_{a}\right)+\beta_{a} \theta_{a}+\left(\eta r \beta_{a} \theta_{b} /\left(r+\gamma_{b}\right)\right)\right]^{2}}{2 \mu_{a} r\left(r+\gamma_{a}\right)^{2}} \\
& -\frac{\left[\delta_{b}\left(r+\gamma_{b}\right)+\beta_{b} \theta_{b}\right]^{2}}{2 \mu_{b} r\left(r+\gamma_{b}\right)^{2}}+\frac{\omega_{a} M+\omega_{b} N}{r} .
\end{aligned}
$$


Substituting equation (85) into equation (83), the optima benefit function can be obtained as

$$
\begin{aligned}
V^{* * *}\left(R_{a}, R_{b}\right)= & \frac{M+N}{r}-\left[\frac{\theta_{a}}{r+\gamma_{a}}-\frac{\lambda \gamma_{a} \theta_{b}}{\left(r+\gamma_{a}\right)\left(r+\gamma_{b}\right)}\right] R_{a}-\frac{\theta_{b}}{r+\gamma_{b}} R_{b}-\frac{\left[\varepsilon_{a}\left(r+\gamma_{a}\right)+\alpha_{a} \theta_{a}+\left(\eta r \alpha_{a} \theta_{b} /\left(r+\gamma_{b}\right)\right)\right]^{2}}{2 \mu_{s} r\left(r+\gamma_{a}\right)^{2}} \\
& -\frac{\left[\varepsilon_{b}\left(r+\gamma_{b}\right)+\alpha_{b} \theta_{b}\right]^{2}}{2 \mu_{s} r\left(r+\gamma_{b}\right)^{2}}-\frac{\left[\delta_{a}\left(r+\gamma_{a}\right)+\beta_{a} \theta_{a}+\left(\eta r \beta_{a} \theta_{b} /\left(r+\gamma_{b}\right)\right)\right]^{2}}{2 \mu_{a} r\left(r+\gamma_{a}\right)^{2}}-\frac{\left[\delta_{b}\left(r+\gamma_{b}\right)+\beta_{b} \theta_{b}\right]^{2}}{2 \mu_{b} r\left(r+\gamma_{b}\right)^{2}} .
\end{aligned}
$$

By deriving (86) and substituting the results into equations (78)-(81), equations (72)-(75) can be obtained.
At this time, the environmental pollution control level of Local-gov A is

$$
\left\{\begin{array}{l}
R_{a}^{* * *}(t)=\frac{1}{\gamma_{a}}\left[\frac{\alpha_{a}}{\mu_{s}}\left(\varepsilon_{a}+\frac{\alpha_{a} \theta_{a}}{r+\gamma_{a}}+\frac{\eta r \alpha_{a} \theta_{b}}{\left(r+\gamma_{a}\right)\left(r+\gamma_{b}\right)}\right)+\frac{\beta_{a}}{\mu_{a}}\left(\delta_{a}+\frac{\beta_{a} \theta_{a}}{r+\gamma_{b}}+\frac{\eta r \beta_{a} \theta_{b}}{\left(r+\gamma_{a}\right)\left(r+\gamma_{b}\right)}\right)-e^{-\gamma_{a} t+D_{a}}\right] \\
R_{a}^{* * *}(0)=R_{a} .
\end{array}\right.
$$
is

The environmental pollution control level of Local-gov B

$$
\left\{\begin{array}{l}
R_{b}^{* *}(t)=\frac{\eta}{\gamma_{b}}\left\{\frac{\alpha_{a}}{\mu_{s}}\left[\varepsilon_{a}+\frac{\alpha_{a} \theta_{a}}{r+\gamma_{a}}+\frac{\eta r \alpha_{a} \theta_{b}}{\left(r+\gamma_{a}\right)\left(r+\gamma_{b}\right)}\right]+\frac{\beta_{a}}{\mu_{a}}\left[\delta_{a}+\frac{\beta_{a} \theta_{a}}{r+\gamma_{a}}+\frac{\eta r \alpha_{a} \theta_{b}}{\left(r+\gamma_{a}\right)\left(r+\gamma_{b}\right)}\right]-e^{-\gamma_{a} t+D_{a}}\right\} \\
+\frac{1}{\gamma_{b}}\left[\frac{\alpha_{b}}{\mu_{s}}\left(\varepsilon_{b}+\frac{\alpha_{b} \theta_{b}}{r+\gamma_{b}}\right)+\frac{\beta_{b}}{\mu_{b}}\left(\delta_{b}+\frac{\beta_{b} \theta_{b}}{r+\gamma_{b}}\right)-e^{-\gamma_{b} t+D_{b}}\right], \\
R_{b}^{* *}(0)=R_{b}, \\
e^{D_{a}}=\frac{\alpha_{a}}{\mu_{s}}\left[\varepsilon_{a}+\frac{\alpha_{a} \theta_{a}}{r+\gamma_{a}}+\frac{\eta r \alpha_{a} \theta_{b}}{\left(r+\gamma_{a}\right)\left(r+\gamma_{b}\right)}\right]+\frac{\beta_{a}}{\mu_{a}}\left[\delta_{a}+\frac{\beta_{a} \theta_{a}}{r+\gamma_{a}}+\frac{\eta r \alpha_{a} \theta_{b}}{\left(r+\gamma_{a}\right)\left(r+\gamma_{b}\right)}\right]-\gamma_{a} R_{a} .
\end{array}\right.
$$

From equations (72)-(75), it can be seen that under the Cooperative model, the optimal efforts of the Cent-gov and Local-gov A no longer involve the distribution ratio of environmental pollution losses, and other influencing factors are consistent with the Nash game model and the Stackelberg model.

\section{Comparative Analysis of Equilibrium Results}

This section compares the equilibrium results, environmental pollution control levels, and environmental pollution losses in the three models, and analyzes whether the Nash game model and the Cooperative model can effectively promote the local governments' environmental pollution control efforts, improve governance level, and reduce environmental pollution losses.

5.1. Analysis of Game Equilibrium Strategy. The comparison of the optimal effort strategies of the Cent-gov and local governments under three models is as follows.

Proposition 4. When $(1 / 3)<\omega \leq 1$, (1) the optimal effort of Local-gov A: $E_{a}^{*}<E_{a}^{* *} \leq E_{a}^{* * *}$; (2) the optimal effort of Localgov B: $E_{b}^{*}<E_{b}^{* *} \leq E_{b}^{* * *}$; (3) the optimal effort of Cent-gov: $E_{a s}^{*}=E_{a s}^{* *} \leq E_{a s}^{* * *}, E_{b s}^{*}=E_{b s}^{* *} \leq E_{b s}^{* * *}$; and (4) the Cent-gov's optimal funding for local governments: $\lambda_{a}^{* *}=\left(E_{a}^{* *}-E_{a}^{*}\right) /$ $E_{a}^{* *}, \lambda_{b}^{* *}=\left(E_{b}^{* *}-E_{b}^{*}\right) / E_{b}^{* *}$. 
Proof. (1) According to equations (7), (39), and (72), we can get

$$
\begin{aligned}
E_{a}^{* *}-E_{a}^{*} & =\frac{3 \omega_{a}-1}{2 \mu_{a}}\left(\delta_{a}+\frac{\beta_{a} \theta_{a}}{r+\gamma_{a}}\right)+\frac{\eta r \beta_{a} \omega_{b} \theta_{b}}{\mu_{a}\left(r+\gamma_{a}\right)\left(r+\gamma_{b}\right)}>0, \\
E_{a}^{* * *}-E_{a}^{* *} & =\frac{1-\omega_{a}}{2 \mu_{a}}\left[\delta_{a}+\frac{\beta_{a} \theta_{a}}{r+\gamma_{a}}+\frac{\eta r \beta_{a} \theta_{b}}{\left(r+\gamma_{a}\right)\left(r+\gamma_{b}\right)}\right]+\frac{\eta r \beta_{a}\left(1-\omega_{b}\right) \theta_{b}}{\left(r+\gamma_{a}\right)\left(r+\gamma_{b}\right)} \geq 0 .
\end{aligned}
$$

Therefore, $E_{a}^{*}<E_{a}^{* *} \leq E_{a}^{* * *}$ is established.

(2) According to equations (8), (40), and (73), we can get

$$
\begin{aligned}
E_{b}^{* *}-E_{b}^{*} & =\frac{3 \omega_{b}-1}{2 \mu_{b}}\left(\delta_{b}+\frac{\beta_{b} \theta_{b}}{r+\gamma_{b}}\right)>0, \\
E_{b}^{* * *}-E_{b}^{* *} & =\frac{1-\omega_{b}}{2 \mu_{b}}\left[\delta_{b}+\frac{\beta_{b} \theta_{b}}{r+\gamma_{b}}+\frac{\eta r \beta_{a} \theta_{b}}{\left(r+\gamma_{a}\right)\left(r+\gamma_{b}\right)}\right]+\frac{\eta r \beta_{b}\left(1-\omega_{b}\right) \theta_{b}}{\mu_{b}\left(r+\gamma_{a}\right)\left(r+\gamma_{b}\right)} \geq 0 .
\end{aligned}
$$

Therefore, $E_{b}^{*}<E_{b}^{* *} \leq E_{b}^{* * *}$ is established.

(3) According to equations (9), (41), and (74), we can get

$$
E_{a s}^{*}=E_{a s}^{* *}
$$

$E_{a s}^{* * *}-E_{a s}^{* *}=\frac{1-\omega_{a}}{\mu_{s}}\left[\varepsilon_{a}+\frac{\alpha_{a} \theta_{a}}{r+\gamma_{a}}+\frac{\eta r \alpha_{a} \omega_{b} \theta_{b}}{\left(r+\gamma_{a}\right)\left(r+\gamma_{b}\right)}\right] \geq 0$.

Therefore, $E_{a s}^{*}=E_{a s}^{* *} \leq E_{a s}^{* * *}$ is established.

According to equations (10), (42), and (75), we can get

$$
\begin{gathered}
E_{b s}^{*}=E_{b s}^{* *}, \\
E_{b s}^{* * *}-E_{b s}^{* *}=\frac{1-\omega_{b}}{\mu_{s}}\left(\varepsilon_{b}+\frac{\alpha_{b} \theta_{b}}{r+\gamma_{b}}\right) \geq 0 .
\end{gathered}
$$

So, $E_{b s}^{*}=E_{b s}^{* *} \leq E_{b s}^{* * *}$.

(4) According to equations (7), (39), and (43), we can get

$$
\begin{aligned}
E_{a}^{* *}-E_{a}^{*} & =\frac{3 \omega_{a}-1}{2 \mu_{a}}\left(\delta_{a}+\frac{\beta_{a} \theta_{a}}{r+\gamma_{a}}\right)+\frac{\eta r \beta_{a} \omega_{b} \theta_{b}}{\mu_{a}\left(r+\gamma_{a}\right)\left(r+\gamma_{b}\right)} \\
& =E_{a}^{* *} \cdot \lambda_{a}^{* *}>0 .
\end{aligned}
$$

According to equations (8), (40), and (44), we can get

$$
E_{b}^{* *}-E_{b}^{*}=\frac{1-\omega_{b}}{2 \mu_{b}}\left[\delta_{b}+\frac{\beta_{b} \theta_{b}}{r+\gamma_{b}}+\frac{\eta r \beta_{a} \theta_{b}}{\left(r+\gamma_{a}\right)\left(r+\gamma_{b}\right)}\right]+\frac{\eta r \beta_{b}\left(1-\omega_{b}\right) \theta_{b}}{\mu_{b}\left(r+\gamma_{a}\right)\left(r+\gamma_{b}\right)}=E_{b}^{* *} \cdot \lambda_{b}^{* *}
$$

The proof is completed.

Proposition 4 shows that when transitioning from the Nash game model to Stackelberg game model, the effort level of the Cent-gov will remain unchanged, while the effort level of Local-gov A and Local-gov B will increase, and the degree of improvement is related to the funding from Cent-gov.

Under the Cooperative game model, all parties have the highest effort level, and the involvement of the Cent-gov has given a positive signal to protect the environment. The local governments tend to actively implement priority strategies and policies promoted by the Cent-gov, otherwise they may be held accountable for inaction. In order to effectively coordinate the allocation of regional environmental pollution control resources, the Cent-gov boosts environmental governance investment through policy leverage.

5.2. Analysis on the Environmental Pollution Control Level. Comparison of environmental pollution control level between Local-gov A and Local-gov B under three models. 
Proposition 5. When $(1 / 3)<\omega \leq 1$, (1) comparison of environmental pollution control levels of Local-gov $A$ is $R_{a}^{*} \leq R_{a}^{* *} \leq R_{a}^{* * *}$ and (2) comparison of environmental pollution control levels of Local-gov $B$ is $R_{b}^{*} \leq R_{b}^{* *} \leq R_{b}^{* * *}$.
Proof. (1) According to equations (34), (70), and (87), we can get

$$
\begin{aligned}
& \left\{\begin{array}{l}
R_{a}^{* *}(t)-R_{a}^{* *}(t)=\frac{\left(1-e^{-\gamma_{a} t}\right) \beta_{a}}{\gamma_{a} \mu_{a}}\left[\frac{3 \omega_{a}-1}{2}\left(\delta_{a}+\frac{\beta_{a} \theta_{a}}{r+\gamma_{b}}\right)+\frac{\eta r \beta_{a} \omega_{b} \theta_{b}}{\left(r+\gamma_{a}\right)\left(r+\gamma_{b}\right)}\right] \geq 0, \\
R_{a}^{*}(0)=R_{a}^{* *}(0),
\end{array}\right. \\
& \left\{\begin{array}{l}
R_{a}^{* * *}(t)-R_{a}^{* *}(t)=\frac{1-e^{-\gamma_{a} t}}{\gamma_{a}}\left\{\frac{\alpha_{a}}{\mu_{s}}\left(1-\omega_{a}\right)\left(\varepsilon_{a}+\frac{\alpha_{a} \theta_{a}}{r+\gamma_{a}}\right)+\frac{\beta_{a}}{2 \mu_{a}}\left[\left(1-\omega_{a}\right)\left(\delta_{a}+\frac{\beta_{a} \theta_{a}}{r+\gamma_{b}}\right)+\frac{\eta r \beta_{a}\left(1-\omega_{b}\right) \theta_{b}}{\left(r+\gamma_{a}\right)\left(r+\gamma_{b}\right)}\right]\right\} \geq 0, \\
R_{a}^{*}(0)=R_{a}^{* *}(0) .
\end{array}\right.
\end{aligned}
$$

So, $R_{a}^{*} \leq R_{a}^{* *} \leq R_{a}^{* * *}$.

$$
\begin{aligned}
& \left\{R_{b}^{* *}(t)-R_{b}^{*}(t)=\frac{1}{\gamma_{b}}\left\{\frac{\eta \beta_{a}\left(1-e^{-\gamma_{a} t}\right)}{\mu_{a}}\left[\frac{3 \omega_{a}-1}{2}\left(\delta_{a}+\frac{\beta_{a} \theta_{a}}{r+\gamma_{a}}\right)+\frac{\eta r \alpha_{a} \omega_{b} \theta_{b}}{\left(r+\gamma_{a}\right)\left(r+\gamma_{b}\right)}\right]+\frac{\left(1+\omega_{b}\right) \beta_{b}\left(1-e^{-\gamma_{b} t}\right)}{2 \mu_{b}}\left(\delta_{b}+\frac{\beta_{b} \theta_{b}}{r+\gamma_{b}}\right)\right\} \geq 0,\right. \\
& R_{b}^{*}(0)=R_{b}^{* *}(0), \\
& \left\{\begin{array}{l}
R_{b}^{* * *}(t)-R_{b}^{* *}(t) \\
=\frac{\eta\left(1-e^{-\gamma_{a} t}\right)}{\gamma_{b}}\left\{\left[\frac{\alpha_{a}\left(1-\omega_{a}\right)}{\mu_{s}}\left(\varepsilon_{a}+\frac{\alpha_{a} \theta_{a}}{r+\gamma_{a}}\right)+\frac{\eta r \alpha_{a} \omega_{b} \theta_{b}}{\left(r+\gamma_{a}\right)\left(r+\gamma_{b}\right)}\right]+\frac{\beta_{a}}{\mu_{a}}\left[\frac{1-\omega_{a}}{2}\left(\delta_{a}+\frac{\beta_{a} \theta_{a}}{r+\gamma_{a}}\right)+\frac{\eta r \alpha_{a}\left(1-\omega_{b}\right) \theta_{b}}{\left(r+\gamma_{a}\right)\left(r+\gamma_{b}\right)}\right]\right\} \\
+\frac{1-e^{-\gamma_{b} t}}{\gamma_{b}}\left[\frac{\alpha_{b}\left(1-\omega_{b}\right)}{\mu_{s}}\left(\varepsilon_{b}+\frac{\alpha_{b} \theta_{b}}{r+\gamma_{b}}\right)+\frac{\beta_{b}\left(1-\omega_{b}\right)}{2 \mu_{b}}\left(\delta_{b}+\frac{\beta_{b} \theta_{b}}{r+\gamma_{b}}\right)\right] \geq 0, \\
R_{b}^{* *}(0)=R_{b}^{* * *}(0) .
\end{array}\right.
\end{aligned}
$$

So, $R_{b}^{*} \leq R_{b}^{* *} \leq R_{b}^{* * *}$.

Proposition 5 shows that compared with the Nash game model, the Stackelberg game model can better promote the improvement of environmental pollution control level for Local-gov A and Local-gov B. In the Cooperative game model, with the maximization of the effort level of the Centgov and local governments, the environmental pollution control level has also reached the highest level.

The Cooperative game model is an effective mechanism to improve the local environmental pollution control level. That is to say, under appropriate circumstances, it is possible for governments to achieve economies of scale and policy spillover effects through cooperation.
5.3. Analysis of Minimum Environmental Pollution Losses. A comparative analysis about environmental pollution losses of Local-gov A, Local-gov B, and Cent-gov under three models.

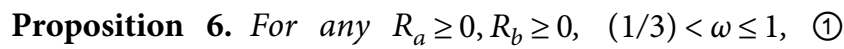
comparison of the optimal environmental pollution losses of Local-gov A: $V_{a}^{*}\left(R_{a}, R_{b}\right) \geq V_{a}^{* *}\left(R_{a}, R_{b}\right)$; (2) comparison of the optimal environmental pollution losses of Local-gov B: $V_{b}^{*}\left(R_{a}, R_{b}\right) \geq V_{b}^{* *}\left(R_{a}, R_{b}\right)$; (3) comparison of the optimal environmental pollution Losses of Cent-gov: $V_{s}^{*}\left(R_{a}, R_{b}\right)$ $\geq V_{s}^{* *}\left(R_{a}, R_{b}\right)$; and (4) comparison of the total 
environmental pollution losses: $V^{*}\left(R_{a}, R_{b}\right) \geq V^{*}\left(R_{a}, R_{b}\right) \quad$ Proof. (1) According to the formulas (30) and (66), we can $\geq V^{* *}\left(R_{a}, R_{b}\right)$.

$$
\begin{aligned}
V_{a}^{*}\left(R_{a}, R_{b}\right)-V_{a}^{* *}\left(R_{a}, R_{b}\right)= & {\left[\frac{\omega_{a} \theta_{a}}{r+\gamma_{a}}-\frac{\lambda \gamma_{a} \theta_{b}}{\left(r+\gamma_{a}\right)\left(r+\gamma_{b}\right)}\right]\left(R_{a}^{* *}-R_{a}^{*}\right)+\frac{\omega_{2} \theta_{b}}{r+\gamma_{b}}\left(R_{b}^{* *}-R_{b}^{*}\right) } \\
& +\frac{\left(1-\omega_{a}\right)\left[\delta_{a}\left(r+\gamma_{a}\right)+\beta_{a} \theta_{a}\right]\left\{\left(3 \omega_{a}-1\right)\left[\delta_{a}\left(r+\gamma_{a}\right)+\beta_{a} \theta_{a}\right]+2 \eta r \beta_{a} \omega_{b} \theta_{b} / r+\gamma_{b}\right\}}{4 \mu_{a} r\left(r+\gamma_{a}\right)^{2}} \geq 0 .
\end{aligned}
$$

So, $V_{a}^{*}\left(R_{a}, R_{b}\right) \geq V_{a}^{* *}\left(R_{a}, R_{b}\right)$.

(2) According to the formulas (31) and (67), we can get

$$
\begin{aligned}
V_{b}^{*}\left(R_{a}, R_{b}\right)-V_{b}^{* *}\left(R_{a}, R_{b}\right)= & {\left[\frac{\omega_{a} \theta_{a}}{r+\gamma_{a}}-\frac{\lambda \gamma_{a} \theta_{b}}{\left(r+\gamma_{a}\right)\left(r+\gamma_{b}\right)}\right]\left(R_{a}^{* *}-R_{a}^{*}\right)+\frac{\theta_{b}}{r+\gamma_{b}}\left(R_{b}^{* *}-R_{b}^{*}\right) } \\
& +\frac{\eta \beta_{a}\left(1-\omega_{b}\right)\left(3 \omega_{a}-1\right) \theta_{b}\left[\delta_{a}\left(r+\gamma_{a}\right)+\beta_{a} \theta_{a}\right]}{2 \mu_{a}\left(r+\gamma_{a}\right)^{2}\left(r+\gamma_{b}\right)}+\frac{\eta^{2} r \alpha_{a} \beta_{a} \omega_{b}\left(1-\omega_{b}\right) \theta_{b}^{2}}{\mu_{a}\left(r+\gamma_{a}\right)^{2}\left(r+\gamma_{b}\right)} \\
& +\frac{\left(1-\omega_{b}\right)\left(3 \omega_{b}-1\right)\left[\delta_{b}\left(r+\gamma_{b}\right)+\beta_{b} \theta_{b}\right]^{2}}{4 \mu_{b} r\left(r+\gamma_{b}\right)^{2}} \geq 0 .
\end{aligned}
$$

So, $V_{b}^{*}\left(R_{a}, R_{b}\right) \geq V_{b}^{* *}\left(R_{a}, R_{b}\right)$.

(3) According to the formulas (32) and (68), we can get

$$
\begin{aligned}
V_{s}^{*}\left(R_{a}, R_{b}\right)-V_{s}^{* *}\left(R_{a}, R_{b}\right)= & {\left[\frac{\omega_{a} \theta_{a}}{r+\gamma_{a}}-\frac{\lambda \gamma_{a} \theta_{b}}{\left(r+\gamma_{a}\right)\left(r+\gamma_{b}\right)}\right]\left(R_{a}^{* *}-R_{a}^{*}\right)+\frac{\theta_{b}}{r+\gamma_{b}}\left(R_{b}^{* *}-R_{b}^{*}\right) } \\
& +\frac{\left\{\left(3 \omega_{a}-1\right)\left[\delta_{a}\left(r+\gamma_{a}\right)+\beta_{a} \theta_{a}\right]+\left(2 \eta r \beta_{a} \omega_{b} \theta_{b} /\left(r+\gamma_{b}\right)\right)\right\}^{2}}{2 \mu_{a}\left(r+\gamma_{a}\right)^{2}\left(r+\gamma_{b}\right)}+\frac{\left(3 \omega_{b}-1\right)^{2}\left[\delta_{b}\left(r+\gamma_{b}\right)+\beta_{b} \theta_{b}\right]^{2}}{8 \mu_{b} r\left(r+\gamma_{b}\right)^{2}}>0 .
\end{aligned}
$$

Thus, $V_{s}^{*}\left(R_{a}, R_{b}\right) \geq V_{s}^{* *}\left(R_{a}, R_{b}\right)$.

(4) $V^{*}\left(R_{a}, R_{b}\right) \geq V^{* *}\left(R_{a}, R_{b}\right)$ is easy to get from (1), (2),

According to the formulas (69) and (86), we can get and (3).

$$
\begin{aligned}
V^{* *}\left(R_{a}, R_{b}\right)-V^{* * *}\left(R_{a}, R_{b}\right)= & {\left[\frac{\omega_{a} \theta_{a}}{r+\gamma_{a}}-\frac{\lambda \gamma_{a} \theta_{b}}{\left(r+\gamma_{a}\right)\left(r+\gamma_{b}\right)}\right]\left(R_{a}^{* * *}-R_{a}^{* *}\right)+\frac{\theta_{b}}{r+\gamma_{b}}\left(R_{b}^{* * *}-R_{b}^{* *}\right) } \\
& +\frac{\left\{\left(1-\omega_{a}\right)\left[\varepsilon_{a}\left(r+\gamma_{a}\right)+\alpha_{a} \theta_{a}\right]+\eta r \alpha_{a}\left(1-\omega_{b}\right) \theta_{b} / r+\gamma_{b}\right\}^{2}}{2 \mu_{s}\left(r+\gamma_{a}\right)^{2}}+\frac{\left(1-\omega_{b}\right)^{2}\left[\varepsilon_{b}\left(r+\gamma_{b}\right)+\alpha_{b} \theta_{b}\right]^{2}}{2 \mu_{s}\left(r+\gamma_{b}\right)} \\
& +\frac{\left\{\left(1-\omega_{a}\right)\left[\delta_{a}\left(r+\gamma_{a}\right)+\beta_{a} \theta_{a}\right]+2 \eta r \beta_{a}\left(1-\omega_{b}\right) \theta_{b} / r+\gamma_{b}\right\}^{2}}{8 \mu_{a}\left(r+\gamma_{a}\right)^{2}}+\frac{\left(1-\omega_{b}\right)^{2}\left[\delta_{b}\left(r+\gamma_{b}\right)+\beta_{b} \theta_{b}\right]^{2}}{2 \mu_{b} r\left(r+\gamma_{b}\right)^{2}} \geq 0 .
\end{aligned}
$$

Thus, $V^{*}\left(R_{a}, R_{b}\right) \geq V^{* *}\left(R_{a}, R_{b}\right) \geq V^{* * *}\left(R_{a}, R_{b}\right)$.

Proposition 6 shows that compared with the Nash game model, the Stackelberg game model promotes the Pareto improvement of all parties, and the environmental pollution losses of all parties are reduced. The Stackelberg game model is better than the Nash game model. From a holistic point of 
TABLE 1: The effort levels of Cent-gov and local governments under the three models.

\begin{tabular}{lcccc}
\hline & $E_{a}$ & $E_{b}$ & $E_{a s}$ & \\
\hline Nash game model & 4.50 & 4.30 & 3.17 & \\
Stackelberg game model & 6.96 & 5.02 & 3.17 & 2.18 \\
Cooperative game model & 9.53 & 7.17 & 6.33 & 2.18 \\
Analysis & $E_{a}^{*}<E_{a}^{* *}<E_{a}^{* * *}$ & $E_{b}^{*}<E_{b}^{* *}<E_{b}^{* * *}$ & $E_{a s}^{*}=E_{a s}^{* *}<E_{a s}^{* * *}$ & 5.44 \\
\hline
\end{tabular}

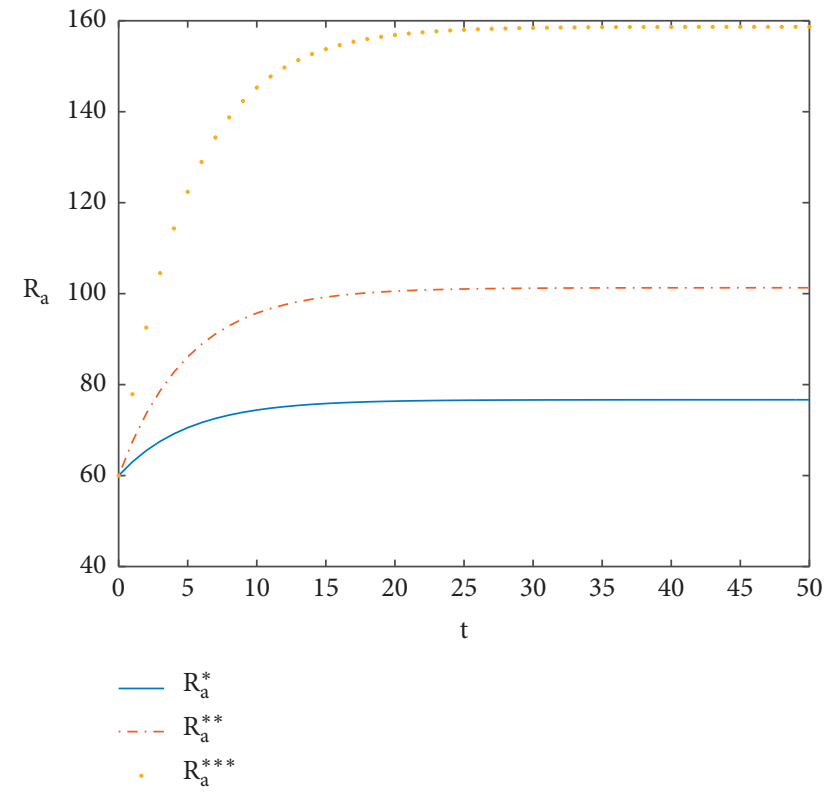

Figure 1: The environmental pollution control level of Local-gov A.

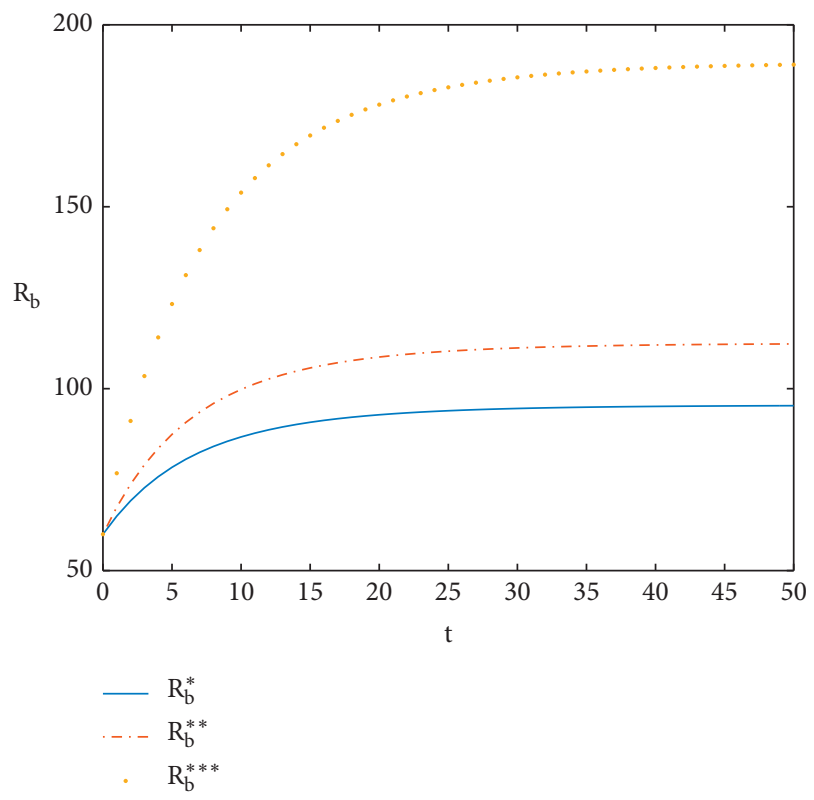

FIGURE 2: The environmental pollution control level of Local-gov B.

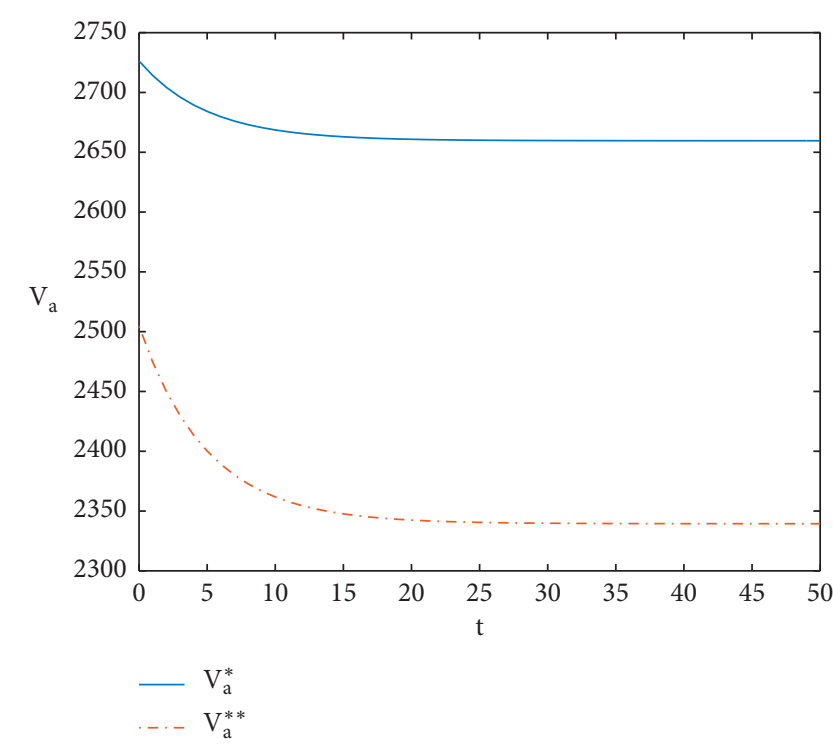

FIgURE 3: Comparison of environmental pollution losses of Localgov A.

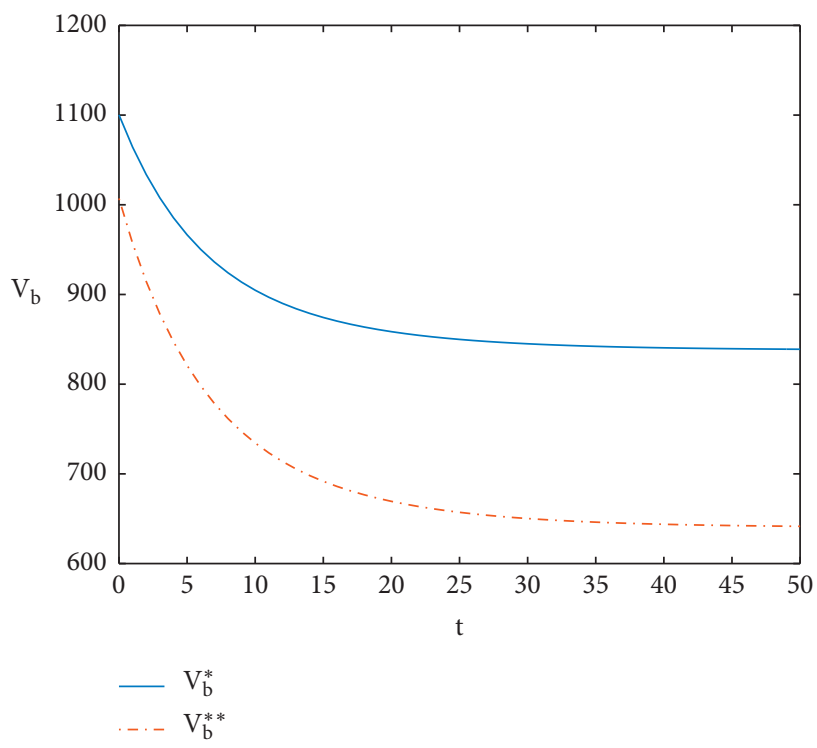

FIGURE 4: Comparison of environmental pollution losses of Localgov B. 
view, if a reasonable environmental pollution losses commitment scheme can be formulated, the Cooperative game model is better than the other two models.

In summary, under the Nash game model, the Cent-gov and local governments as subjects with relatively independent interests and responsibilities choose the optimal effort level from the perspective of minimizing their own environmental losses, without considering the overall interests of the region. In order to improve the local environment and promote local economic development, local governments will take proactive actions to control environmental pollution. However, due to the division of administrative power and the positive externality of environmental governance, local governments have a negative attitude towards matters involving multiple administrative regions. The environmental pollution emergency linkage benefits are almost negligible. In the Stackelberg game model, the intervention of the Cent-gov can effectively solve the market failure caused by the externalities of environmental pollution, optimize resource allocation to a certain extent, and effectively reduce environmental pollution losses. By formulating a clear financial subsidy system, the central government strengthens financial support to local governments, which can effectively reduce the self-interested behavior of local governments, consolidate the trust between the local government and the central government, and promote more effective cooperation between the two parties. The Cooperative game model, which aims at minimizing the environmental pollution losses, is the best choice for Cent-gov, Local-gov A, and Local-gov B during environmental governance, and can effectively control the environmental pollution losses.

\section{Numerical Analyses}

Under the three modes, the optimal effort level, environmental pollution control level, and environmental pollution losses of Local-gov A, Local-gov B, and Cent-gov depend on the selection of various parameters in the models. If we assume that $R_{a}(0)=R_{b}(0)=60$, in the models, discount rate $r=0.05$, and the pollution losses distribution ratios are $\omega_{1}=0.5, \omega_{2}=0.4, \quad \mu_{s}=3, \mu_{a}=2, \mu_{b}=2, \quad \alpha_{a}=2, \alpha_{b}=1$, $\beta_{a}=2, \beta_{b}=1$, the attenuation rates of environmental pollution control level are: $\gamma_{a}=0.2, \gamma_{b}=0.1, \varepsilon_{a}=3, \varepsilon_{b}=3$, $\delta_{a}=2, \delta_{b}=1, \theta_{a}=2, \theta_{b}=2, \quad \eta=0.2$. Under the three models, the effort levels of Cent-gov and local governments are shown in Table 1.

In the Nash game model, environmental pollution control efforts of the Cent-gov and local governments are always the lowest; in the Stackelberg game model, the environmental pollution control effort of the Cent-gov is consistent with that in the Nash game model. However, the environmental pollution control efforts of Local-gov A and Local-gov B have improved to some extent; in the Cooperative game model, the efforts of all parties have significantly improved and reach the highest state, which are consistent with the conclusions of Proposition 4.

In the Nash game model, the environmental pollution control level of Local-gov A is $R_{a}^{*}=76.67-16.67 e^{-0.2 t}$, the

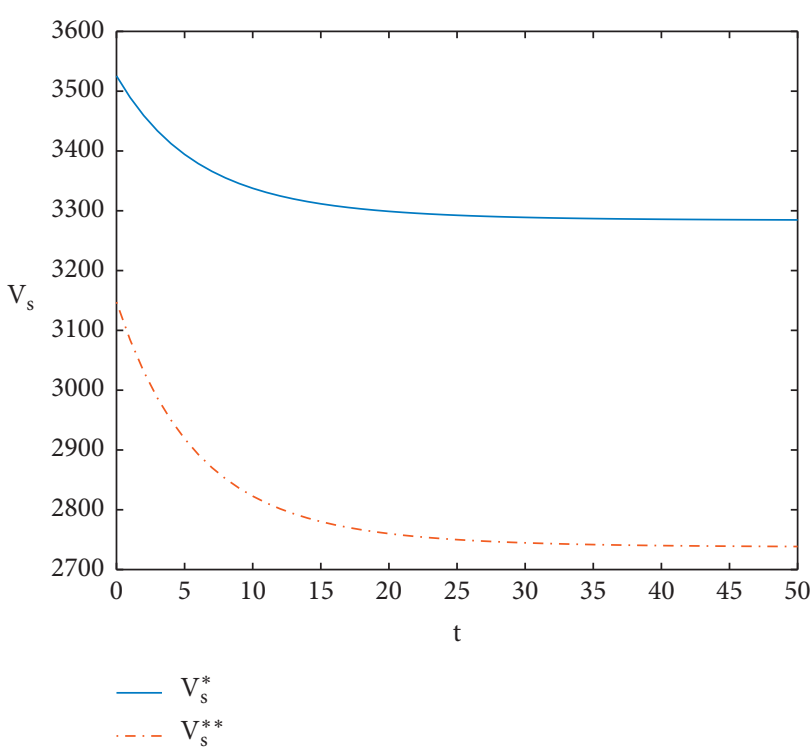

Figure 5: Comparison of environmental pollution losses of Centgov.

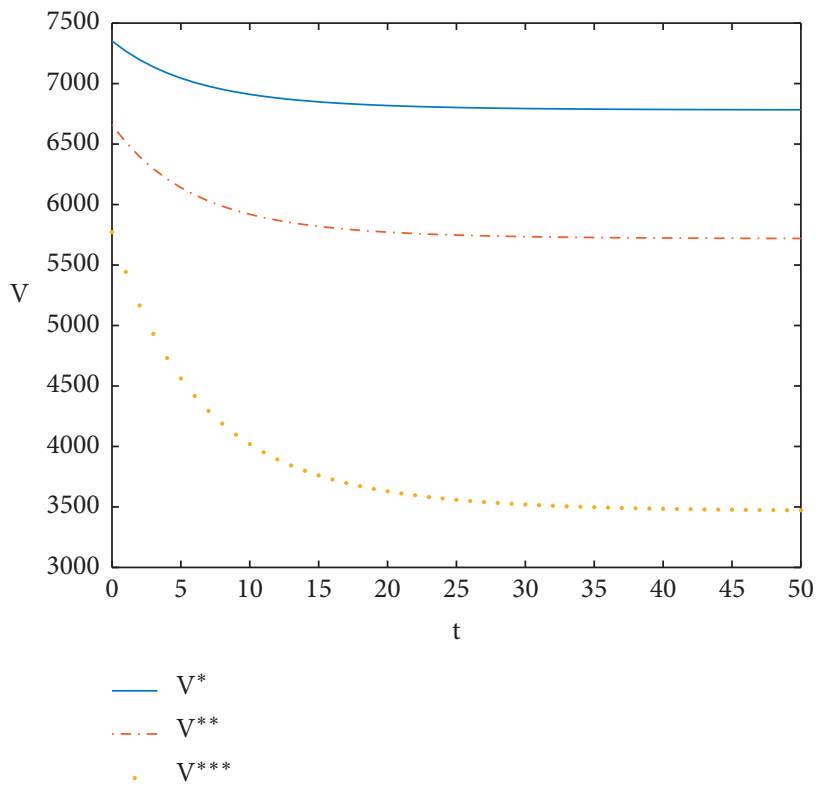

Figure 6: Comparison of total environmental pollution losses in the region.

environmental pollution control level of Local-gov B is $R_{b}^{*}=95.44-18.67 e^{-0.2 t}-16.78 e^{-0.1 t}$, the minimum environmental pollution losses of Local-gov $\mathrm{A}$ is $V_{a}^{*}=$ $2966.31-4 R_{a}$, the minimum environmental pollution losses of Local-gov B is $V_{b}^{*}=1503.4+1.28 R_{a}-8 R_{b}$, the minimum environmental pollution losses of Cent-gov is $V_{s}^{*}=4034.32$ $-3.15 R_{a}-5.33 R_{b}$, the total environmental pollution losses in the region is $V^{*}=8504.04-5.87 R_{a}-13.33 R_{b}$; in the Stackelberg game model, the environmental pollution control level of Local-gov A is $R_{a}^{* *}=101.3-41.3 e^{-0.2 t}$, the environmental pollution control level of Local-gov $B$ is $R_{b}^{* *}=112.46-28.52 e^{-0.2 t}-23.94 e^{-0.1 t}$, the minimum 
environmental pollution losses of Local-gov $\mathrm{A}$ is $V_{a}^{* *}=$ $2744.61-4 R_{a}$, the minimum environmental pollution losses of Local-gov B is $V_{b}^{* *}=1410.24+1.28 R_{a}-8 R_{b}$, the minimum environmental pollution losses of Cent-gov is $V_{s}^{* *}=3656.16-3.15 R_{a}-5.33 R_{b}$, the total environmental pollution losses in the region is $V^{* *}=7811.01-5.87 R_{a}$ $-13.33 R_{b}$; in the Cooperative game model, the environmental pollution control level of Local-gov $A$ is $R_{a}^{* * *}=158.67-98.67 e^{-0.2 t}$, the environmental pollution control level of Local-gov $\mathrm{B}$ is $R_{b}^{* * *}=189.58-$ $51.47 e^{-0.2 t}-78.11 e^{-0.1 t}$, the total environmental pollution losses in the region is $V^{* * *}=6923.59-5.87 R_{a}-13.33 R_{b}$.

The trends of environmental pollution control levels of Local-gov A and Local-gov B are shown in Figures 1 and 2. Under the three models, the improvement of environmental pollution control level tends to be stable over time. In Nash game model, the environmental pollution control levels are the lowest; in the Stackelberg game model, the environmental pollution control levels have been improved; and in the Cooperative game model, the environmental pollution control levels have always been higher than the other two models, which are consistent with the conclusions of Proposition 5.

Before the improvement of environmental pollution control level stabilizes, the growth rate of the environmental pollution control level in the Nash game model is the slowest, the growth rate of the Stackelberg game model is medium, and the growth rate of the Cooperative game model is the fastest.

In the three models, the trend of environmental pollution losses of Local-gov A, Local-gov B, Cent-gov, and the region over time is shown in Figures 3-6. Under the Stackelberg game model, the environmental pollution losses of Local-gov A, Local-gov B, and Cent-gov increase over time and tend to be stable, and are always lower than that under the Nash game model; The regional total environmental pollution losses under the Cooperative game model is always lower than the environmental pollution losses of Local-gov A under the Nash game model and the Stackelberg game model, which are consistent with the conclusions of Proposition 6.

\section{Conclusions and Policy Recommendations}

7.1. Conclusions. This paper constructs two functions about the environmental pollution control level and environmental pollution losses, which are used to study the environmental pollution control problem between the Cent-gov and the two local governments. The local governments improve environmental pollution control levels through their own efforts and the Cent-gov's funding and seek to minimize environmental pollution losses in an infinite time zone.

This paper constructs a tripartite differential game model, and considers the equilibrium results, environmental pollution control level, and minimum pollution losses of the Cent-gov and the two local governments in the three models, and then carries out the comparative analysis. The result shows the following:
(1) In three situations, the cost coefficient of all governments, the attenuation coefficient of environmental pollution control level, and the discount rate are inversely proportional to the environmental pollution effort; the government's ability to influence and govern are directly proportional to environmental pollution effort.

(2) In the process of environmental pollution control, the parties put in more efforts in the Cooperative game model than the other two models.

(3) From the perspective of environmental pollution control level and regional total pollution losses, the Cooperative game model is superior to the Nash game model and the Stackelberg model, which can achieve the Pareto optimal of the system.

(4) This paper quantitatively analyzes the relationship between central government funding and the proportion of environmental pollution losses, the effort level of local government, and the environmental pollution control level. The Cent-gov's special subsidy can effectively encourage local governments to put in more environmental pollution control efforts and reduce all parties' pollution losses and achieve Pareto optimal.

\subsection{Policy Recommendations}

(1) Reform the fiscal decentralization system and improve the performance evaluation standards of local governments. For a long time, the performance evaluation standards of local governments have always been around GDP growth. Local governments have paid more attention to local economic development and paid less attention to nonproductive expenditures such as environmental protection. Expenditure on economic construction has greatly squeezed out expenditure on energy conservation and environmental protection, and ecological and environmental protection has been neglected in the process of economic development. Therefore, the Cent-gov should completely abandon the "GDPonly" performance evaluation system, accelerate the inclusion of environmental protection and sustainable development indicators into the evaluation system, promote the coordinated and healthy development of the economy and the environment, and achieve green fiscal competition.

(2) Strengthen the cooperation of governments at all levels to promote the equalization of local environmental governance. The environmental protection requires a large amount of capital investment, and only relying on local fiscal revenue to ensure the growth of environmental protection expenditure cannot be sustained. The research results of this paper show that government special subsidies can effectively encourage local governments to take more environmental protection efforts and reduce environmental pollution losses 
from all parties. Therefore, the Cent-gov must strengthen special transfer payments to local governments for environmental protection, and use special funds to solve the contradiction between the local government's environmental protection needs and the shortage of financial funds, and establish a horizontal transfer payment system. Because environmental pollution is transboundary and environmental protection has positive externality, the central government should formulate an environmental compensation mechanism between local governments, and regions that benefit from environmental protection should provide compensation to regions that provide environmental protection.

(3) Improve the transparency of fiscal expenditures and strengthen social supervision and central supervision. On the one hand, the Cent-gov should further strengthen fiscal transparency, regulate fiscal revenue and expenditure, harden budget constraints, and mobilize social supervision; on the other hand, it should emphasize fiscal incentives for green investment in enterprises, and use policy guidance to help prevent and control environmental pollution. Through local finance, companies that invest in environmental protection are provided with indirect tax reductions, and companies that use pollutionfree equipment in the production process directly enjoy tax credits, so that companies can develop advanced technologies and factories to reduce environmental pollution.

With the development of global economic integration, environmental pollution and destruction are not only a domestic cross-administrative regional cooperative governance problem but also an international cross-border cooperative governance problem [39]. For example, a large amount of greenhouse gas emissions have caused global warming. Water pollution of the Rhine River caused widespread death of aquatic life in coastal countries. The environmental governance investment, as a typical international public goods supply, has significant positive externality, and the benefits and costs brought by it are seriously unequal, which makes it difficult to achieve international cooperation and international coordination. Therefore, certain measures need to be taken to promote international government cooperation. We can start from the following aspects:

(1) Enhance the linkage effect of ecological cooperation between countries. All countries should continuously improve their domestic ecological protection policies and institutional systems, provide policy support for environmental protection, and actively participate in the construction of ecological civilization; all countries should formulate mutually supportive policy systems and carry out in-depth cooperation to reduce national barriers toward the implementation of environmental protection policies; strengthen the role of international organizations in providing public goods, expand the sources of environmental protection funds, and ensure the adequacy and continuity of capital investment.

(2) Call on all countries to strengthen ecological and environmental protection. On the one hand, international organizations represented by the United Nations should actively promote the global action concept of ecological civilization construction, continuously create an atmosphere of common action and common governance around the world, and increase the enthusiasm and initiative of each country to participate in environmental changes; on the other hand, developed countries should play an exemplary role, follow historical logic, actively assume common but differentiated responsibilities, and work with developing countries to solve the ecological and environmental dilemmas faced by mankind.

(3) Improve the cost-sharing mechanism and compensation mechanism for environmental protection. The benefits of environmental protection investment have the characteristics of lagging and being risky, and may not be able to obtain benefits in the short term. Then, other countries should share and compensate the countries that invested in the early stage, so as to form a long-term cooperative relationship. The "free rider" problem in the supply of international public goods can be solved, and the "prisoner's dilemma" can be transformed into a cooperative game.

The shortcomings of this paper are the following three points: Firstly, this paper only considers the intergovernmental relationship between the Cent-gov and the two local governments. In reality, the process of regional environmental pollution control will involve more than two local governments; secondly, this paper only considers the impact of major participants' efforts and environmental pollution control levels on environmental pollution losses; however, in reality, many factors are contributing to the losses due to environmental pollution, and they are mutually connected and also affected; finally, for the convenience of solving, this paper assumes that all parameters in the models are not related to time, the subsequent research can use a differential game model to solve the nondegenerate problem.

\section{Data Availability}

The data used to support the findings of this study are included within the article.

\section{Conflicts of Interest}

The authors declare that there are no conflicts of interest.

\section{Authors' Contributions}

All authors contributed equally to this paper. 


\section{Acknowledgments}

This work was sponsored by Art Science Planning Project of Tianjin (C20014).

\section{References}

[1] C. Meng, X. Song, K. Tian, B. Ye, and T. Si, "Spatiotemporal variation characteristics of water pollution and the cause of pollution formation in a heavily polluted river in the upper Hai river," Journal of Chemistry, vol. 2020, Article ID 6617227, 15 pages, 2020.

[2] Z. Yin, L Zhu, S Li et al., "A comprehensive review on cultivation and harvesting of microalgae for biodiesel production: environmental pollution control and future directions," Bioresource Technology, vol. 301, Article ID 122804, 2020.

[3] L. Zhao and S. Jin, "Research on the impact of ecological civilization construction on environmental pollution control in China-based on differential game theory," Discrete Dynamics in Nature and Society, vol. 2021, Article ID 5552069, 12 pages, 2021.

[4] S. De, W. Wang, Y. Zhou et al., "Analyzing environmental impact of large-scale events in public spaces with cross-domain multimodal data fusion," Computing, vol. 4, pp. 1-23, 2021.

[5] R. S. Chirinko and D. J. Wilson, "Tax competition among U.S. States: racing to the bottom or riding on a seesaw?" Journal of Public Economics, vol. 155, pp. 147-163, 2017.

[6] J. Perdiguero and J. L. Jiménez, "Sell or not sell biodiesel: local competition and government measures," Renewable and Sustainable Energy Reviews, vol. 15, no. 3, pp. 1525-1532, 2011.

[7] C. Xu, "The fundamental institutions of China's reforms and development," Journal of Economic Literature, vol. 49, no. 4, pp. 1076-1151, 2011.

[8] H. Lin, S. X. Zeng, H. Y. Ma, G. Y. Qi, and V. W. Y. Tam, “Can political capital drive corporate green innovation? Lessons from China," Journal of Cleaner Production, vol. 64, pp. 6372, 2014

[9] T. R. Kubick and A. N. S. Masli, "Firm-level tournament incentives and corporate tax aggressiveness," Journal of Accounting and Public Policy, vol. 35, pp. 66-83, 2016.

[10] J. Yu, L.-A. Zhou, and G. Zhu, "Strategic interaction in political competition: evidence from spatial effects across Chinese cities," Regional Science and Urban Economics, vol. 57, pp. 23-37, 2016.

[11] S. Hong and S. Lee, "Adaptive governance, status quo bias, and political competition: why the sharing economy is welcome in some cities but not in others," Government Information Quarterly, vol. 35, no. 2, pp. 283-290, 2018.

[12] X. Shi and T. Xi, "Race to safety: political competition, neighborhood effects, and coal mine deaths in China," Journal of Development Economics, vol. 131, pp. 79-95, 2018.

[13] J. Gang, K. Shen, and L. Jian, "Interjurisdiction political competition and green total factor productivity in China: an inverted-U relationship," China Economic Review, vol. 61, Article ID S1043951X18301287, 2020.

[14] Y. Deng, D. You, and J. Wang, "Optimal strategy for enterprises' green technology innovation from the perspective of political competition," Journal of Cleaner Production, vol. 235, pp. 930-942, 2019.

[15] F. Wu, "Local government competition and industrial structure convergence," New Frontiers in Regional Science: Asian Perspectives, vol. 5, pp. 159-184, 2015.
[16] Deng, Zhang, Ahmad, and Draz, "Local government competition, environmental regulation intensity and regional innovation performance: an empirical investigation of Chinese provinces," International Journal of Environmental Research and Public Health, vol. 16, no. 12, p. 2130, 2019.

[17] R. J. Fairchild, “The manufacturing sector's environmental motives: a game-theoretic analysis," Journal of Business Ethics, vol. 79, no. 3, pp. 333-344, 2008.

[18] X. Qiu and J. Yang, "Government role in the coordinated mechanism for River Basin water quality control," Energy Procedia, vol. 5, pp. 337-342, 2011.

[19] M. Z. Mir, B. Chatterjee, and R. Taplin, "Political competition and environmental reporting," Asian Review of Accounting, vol. 23, no. 1, pp. 17-38, 2015.

[20] X.-J. Li and N. He, "Regional competition environmental tax and enterprise green technology innovation," China Population, Resources and Environment, vol. 28, pp. 73-81, 2018.

[21] T. Eichner and R. Pethig, "Strategic pollution control and capital tax competition," Journal of Environmental Economics and Management, vol. 94, pp. 27-53, 2019.

[22] Y. Ren, "Main problems and future prospects of environmental supervision in Guangdong-Hong Kong-Macau grand Bay area," Chinese Studies, vol. 9, no. 2, pp. 53-64, 2020.

[23] J. Tang and F. Qin, "Analysis of the impact of local government competition on green total factor productivity from the perspective of factor market distortion-based on the three-stage DEA model," 2021.

[24] H. Cremer, P. D. Donder, and F. Gahvari, "Political competition within and between parties: an application to environmental policy," Journal of Public Economics, vol. 92, pp. 532-547, 2005.

[25] Y. Qi and L. Zhang, "Local environmental enforcement constrained by central-local relations in China," Environmental Policy and Governance, vol. 24, no. 3, pp. 216-232, 2014.

[26] I. Fedyukin and Igor, "“Westernizations" from Peter I to Meiji: war, political competition, and institutional change," Theory and Society, vol. 47, no. 2, pp. 207-231, 2018.

[27] X. Yu, "Transboundary water pollution management lessons learned from river basin management in China, Europe and The Netherlands," Utrecht Law Review, vol. 7, no. 1, pp. 188-203, 2011.

[28] D. O. Kim, A. Dinart, and A. Mckinney, "Transboundary water management: can issue linkage help mitigate externalities?" International Game Theory Review, vol. 14, pp. 35-85, 2012.

[29] L. Fernandez, "Transboundary water institutions in action," Water Resources and Economics, vol. 1, pp. 20-35, 2013.

[30] C. Li, D. Sun, X. Xie, and J. Xue, "Cooperation control strategies for China's cross-region pollution in a lake basin based on green reduction cost," Journal of the Air \& Waste Management Association, vol. 66, no. 5, pp. 482-491, 2016.

[31] J. K. Brueckner and K. Jan, "Strategic interaction among governments: an overview of empirical studies," International Regional Science Review, vol. 26, no. 2, pp. 175-188, 2003.

[32] J. D. Wilson and R. H. Gordon, "Expenditure competition," Journal of Public Economic Theory, vol. 5, pp. 1-20, 2010.

[33] H. Xu, H. Yin, and Y. Guo, "Fiscal decentralization, local government competition and farmland conversion in China: the co-integration analysis and the GMM estimation based on the inter-provincial panel data," Agricultural Economics, vol. 59, pp. 420-429, 2013. 
[34] D. Becker and M. Rauscher, "Fiscal competition and growth when capital is imperfectly mobile," The Scandinavian Journal of Economics, vol. 115, no. 1, pp. 211-233, 2013.

[35] D. Prud'homme, "Dynamics of China's provincial-level specialization in strategic emerging industries," Research Policy: A Journal Devoted to Research Policy, Research Management and Planning, vol. 45, pp. 98-115, 2016.

[36] J. Skovgaard, "The role of finance ministries in environmental policy making: the case of European union emissions trading system reform in Denmark, Germany and The Netherlands," Environmental Policy and Governance, vol. 27, no. 4, pp. 351-364, 2017.

[37] S. Ercolano and O. Romano, "Spending for the environment: general government expenditure trends in Europe," Social Indicators Research: An International and Interdisciplinary, vol. 138, no. 3, pp. 1145-1169, 2018.

[38] A. Bazavan, "Chinese government's shifting role in the national innovation system," Technological Forecasting and Social Change, vol. 148, Article ID 119738, 2019.

[39] L. Zhao and C. O. A. Otoo, "Stability and complexity of a novel three-dimensional environmental quality dynamic evolution system," Complexity, vol. 2019, Article ID 3941920, 11 pages, 2019. 